\title{
Graphene-Coated Nanowire Waveguides and Their Applications
}

\author{
Da Teng ${ }^{1, *} \mathbb{0}$, Kai Wang ${ }^{2, *}$ and ${\mathrm{Zhe} \mathrm{Li}^{3}}^{3}$ \\ 1 School of Physics and Electronic Engineering, Zhengzhou Normal University, Zhengzhou 450044, China \\ 2 Key Laboratory of Infrared Imaging Materials and Detectors, Shanghai Institute of Technical Physics, \\ Chinese Academy of Sciences, Shanghai 200083, China \\ 3 College of Sciences, Shanghai University, Shanghai 200444, China; lizhecn@shu.edu.cn \\ * Correspondence: tengda@zznu.edu.cn (D.T.); wangkai@mail.sitp.ac.cn (K.W.); \\ Tel.: +86-0371-6550-2273 (D.T.); +86-021-2505-1475 (K.W.)
}

Received: 14 January 2020; Accepted: 26 January 2020; Published: 28 January 2020

\begin{abstract}
In recent years, graphene-coated nanowires (GCNWs) have attracted considerable research interest due to the unprecedented optical properties of graphene in terahertz $(\mathrm{THz})$ and mid-infrared bands. Graphene plasmons in GCNWs have become an attractive platform for nanoscale applications in subwavelength waveguides, polarizers, modulators, nonlinear devices, etc. Here, we provide a comprehensive overview of the surface conductivity of graphene, GCNW-based plasmon waveguides, and applications of GCNWs in optical devices, nonlinear optics, and other intriguing fields. In terms of nonlinear optical properties, the focus is on saturable absorption. We also discuss some limitations of the GCNWs. It is believed that the research of GCNWs in the field of nanophotonics will continue to deepen, thus laying a solid foundation for its practical application.
\end{abstract}

Keywords: graphene-coated nanowires; graphene plasmons; mid-infrared waves; waveguide

\section{Introduction}

The manipulation of light-matter interaction at subwavelength scale using surface plasmons (SPs) [1-3], which could confine the electromagnetic fields into regions far below the diffraction limit [4], has been widely exploited due to various applications [5-8]. However, the inherent shortages of the metallic structures hinder their application in some plasmonic devices.

Graphene [9], a two-dimensional (2D) carbon material, was proved to be an alternate material that could excite SPs in the mid and far-infrared bands owing to its unprecedented properties such as the tunability, extremely strong modal field confinement, and huge field enhancement [10-12]. Therefore, lots of graphene-based novel optical devices, including waveguides [13-19], modulators [20,21], switches [22-24], polarizers [25,26], sensors [27,28], antenna [29], etc., have been developed, and the research of graphene plasmonics has promoted the development of nanophotonics. Particularly, the GCNWs [30-35] have aroused lots of research interest, and the wide applications of GCNWs in photonic devices have become one of the research hot spots in recent years. It is worth mentioning that although there already exists a review of graphene plasmonic waveguides [36], not enough emphasis is laid on the GCNWs and their applications.

Here, we review the latest research status of photonic devices based on GCNWs with a particular emphasis on various kinds of GCNW waveguides and their applications in photonic devices, nonlinear devices, lasers, and other new interesting fields. In order to get a better understanding of the condition of the graphene supporting surface plasmon modes, we first look back at the surface conductivity of graphene. We hope that the GCNWs will play a key role in future development of photonic devices. 


\section{Surface Conductivity of Graphene}

The optical properties of graphene are described by its complex surface conductivity $\sigma_{\mathrm{g}}=\sigma_{\mathrm{r}}+$ $j \sigma_{\mathrm{i}}$, where $\sigma_{\mathrm{r}}$ and $\sigma_{\mathrm{i}}$ represent the real and imaginary parts, respectively. For a graphene layer with thickness of $d$, the equivalent complex permittivity is given as [12,37]:

$$
\varepsilon_{\mathrm{g}}=\varepsilon_{0}-\frac{\sigma_{\mathrm{i}}}{\omega d}+\frac{\sigma_{\mathrm{r}}}{\omega d} j
$$

where $\varepsilon_{0}$ is the vacuum permittivity and $\omega$ is the angular frequency of the light. The real (imaginary) part of $\varepsilon_{\mathrm{g}}$ is related to the imaginary (real) part of $\sigma_{\mathrm{g}}$. For a sufficiently small $d$, the real part could be approximately written as

$$
\operatorname{Re}\left(\varepsilon_{\mathrm{g}}\right) \approx-\sigma_{\mathrm{i}} /(\omega d)
$$

It can be seen that the real part of equivalent permittivity for the $d$-thick graphene layer can be positive or negative decided by the sign of the imaginary part of the graphene conductivity. When $\sigma_{\mathrm{i}}>0$, i.e., $\operatorname{Re}\left(\varepsilon_{\mathrm{g}}\right)<0$, the graphene layer shows "metallic" properties, and could support a transverse-magnetic (TM) surface plasmon mode [1].

Further, the relative equivalent complex permittivity of graphene can be calculated by using $\varepsilon_{\mathrm{g}}=1+j \sigma_{\mathrm{g}} /\left(\varepsilon_{0} \omega d\right)$ [12], where $d=0.335 \mathrm{~nm}$ is the thickness of monolayer graphene [38,39]. Within the random-phase approximation, the dynamic optical response of graphene can be derived from the Kubo's formula consisting of the interband and intraband contributions in the infrared ranges, that is $\sigma_{\mathrm{g}}=\sigma_{\text {intra }}+\sigma_{\text {inter }}$, and the surface conductivity of graphene is given as [40]:

$$
\begin{gathered}
\sigma_{\mathrm{g}}=\sigma_{\text {intra }}+\sigma_{\text {inter }} \\
\sigma_{\text {intra }}=\frac{2 j e^{2} k_{B} T}{\pi \hbar^{2}(\omega+j / \tau)} \ln \left[2 \cosh \left(\frac{\mu_{\mathrm{c}}}{2 k_{B} T}\right)\right] \\
\sigma_{\text {inter }}=\frac{e^{2}}{4 \hbar}\left[\frac{1}{2}+\frac{1}{\pi} \arctan \left(\frac{\hbar \omega-2 \mu_{\mathrm{c}}}{2 k_{B} T}\right)-\frac{j}{2 \pi} \ln \frac{\left(\hbar \omega+2 \mu_{\mathrm{c}}\right)^{2}}{\left(\hbar \omega-2 \mu_{\mathrm{c}}\right)^{2}+\left(2 k_{B} T\right)^{2}}\right]
\end{gathered}
$$

where $\tau$ is the electron relaxation time, $T=300 \mathrm{~K}$ is the temperature, $\mu_{\mathrm{c}}$ is the chemical potential of the graphene, $\hbar$ is the reduced plank constant, $k_{B}$ is the Boltzmann's constant, and $e$ is the charge of the electron. In the calculation, the graphene could be either treated as a thin layer or surface current. For convenience, graphene is usually modelled as an electric field-induced surface current $\mathbf{J}=\sigma_{\mathrm{g}} \mathbf{E}$ on the surface of nanowire.

The tunability of the graphene is of great importance for practical applications in photonic devices. As we have shown before, the equivalent permittivity of graphene can be positive or negative depending on the sign of the imaginary part of the graphene conductivity. Hence, the imaginary part of the graphene conductivity $\sigma_{\mathrm{i}}$ determines the propagation of transverse-electric (TE) or TM modes. Clearly, when $\sigma_{\mathrm{i}}>0\left(\operatorname{Re}\left(\varepsilon_{\mathrm{g}}\right)<0\right)$, the graphene layer could support a TM SP mode. On the contrary, when $\sigma_{\mathrm{i}}<0$, the graphene layer could support a TE mode.

Figure 1 depicts the surface conductivity of the graphene as functions of frequency and chemical potential. Here, we focus on the terahertz and mid-infrared bands, thus $f_{0}$ ranges from $1 \mathrm{THz} 100 \mathrm{THz}$. The graphene conductivity values are all normalized by $\sigma_{0}=e^{2} / \hbar$. One can see from Figure 1 a that for the frequencies considered here, $\sigma_{\mathrm{i}}$ is always above 0 , and TM modes can be propagated. The interband and intraband contributions of the real parts of the graphene conductivity $\operatorname{Re}\left(\sigma_{\mathrm{g}}\right)$ are shown in Figure $1 \mathrm{~b}$. It indicates that the intraband contribution dominates in the low frequency range, that is $\sigma_{\mathrm{g}} \approx \sigma_{\text {inter }}$. While at higher frequency range $\left(f_{0}>48 \mathrm{THz}\right)$, the interband contribution takes over. Therefore, the intraband (interband) contribution is responsible for loss at low (high) frequencies. In Figure 1c, we plot the real and imaginary parts of $\sigma_{\mathrm{g}}$ with respect to $\mu_{\mathrm{c}}$. It is found that at very small $\mu_{\mathrm{c}}, \sigma_{\mathrm{r}}$ is higher than $\sigma_{\mathrm{i}}$. Note that by increasing $\mu_{\mathrm{c}}, \sigma_{\mathrm{r}}$ decreases to nearly zero, which indicates that a significant reduction in loss can be achieved by using high chemical potential values. For $\mu_{\mathrm{c}}>0.24 \mathrm{eV}$, 
$\sigma_{\mathrm{i}}>0$, and TM SP modes can be supported in the considered range. The interband and intraband contributions of the real parts of the graphene conductivity with respect to $\mu_{\mathrm{c}}$ are shown in Figure $1 \mathrm{~d}$. It shows that the interband transition drastically reduces as $\mu_{\mathrm{c}}$ increases, and the intraband contribution dominates at higher $\mu_{\mathrm{c}}$. Also, intraband and interband contributions are at the same level, which suggests one can reduce the modal loss easily by enhancing $\mu_{\mathrm{c}}$.

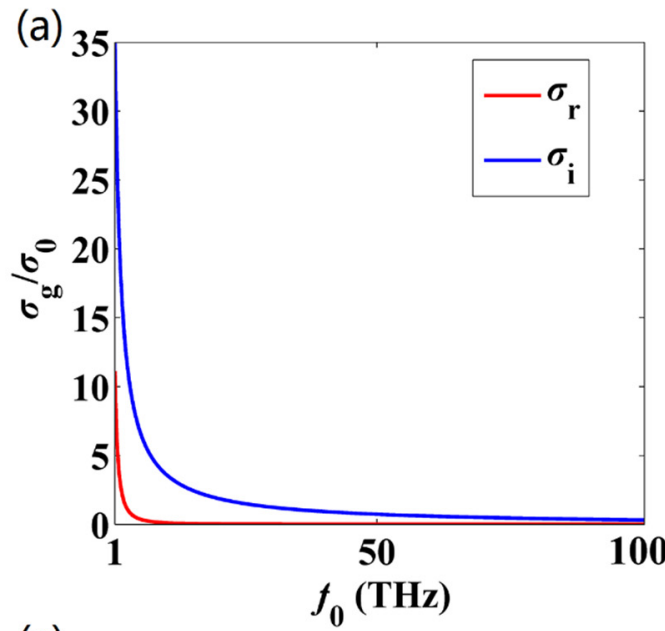

(c)

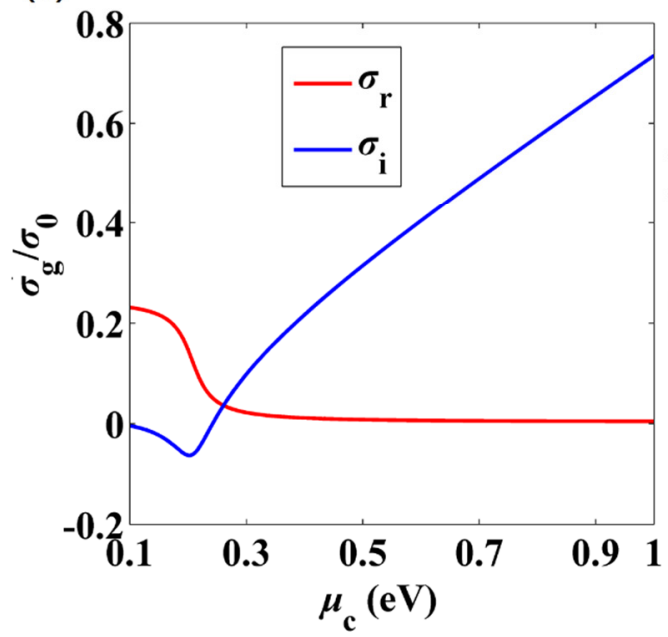

(b)

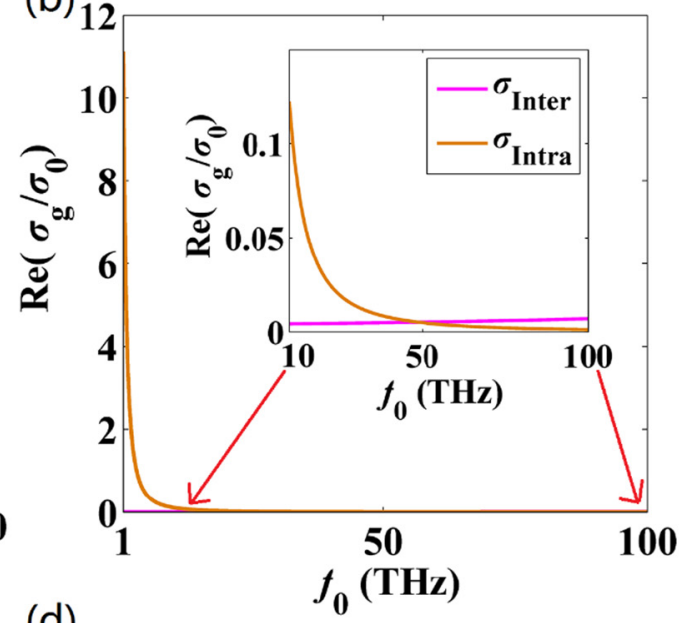

(d)

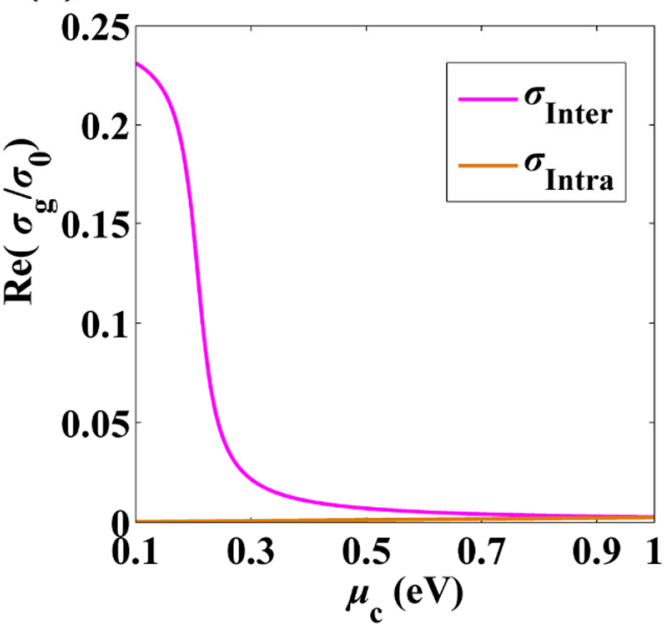

Figure 1. The graphene conductivity $\sigma_{\mathrm{g}}$, normalized by $\sigma_{0}=e^{2} / \hbar$, as functions of the frequency and chemical potential, calculated from Equation (3). (a) Real and imaginary parts of $\sigma_{\mathrm{g}}$ with respect to frequency. (b) Interband and intraband contributions of the real parts of the graphene conductivity with respect to frequency. (c) Real and imaginary parts of $\sigma_{\mathrm{g}}$ with respect to $\mu_{\mathrm{c}}$. (d) Interband and intraband contributions of the real parts of the graphene conductivity with respect to $\mu_{\mathrm{c}}$. The chemical potential $\mu_{\mathrm{c}}$ is $0.5 \mathrm{eV}$ for $(\mathbf{a}, \mathbf{b})$, and the frequency is $20 \mathrm{THz}$ for $(\mathbf{c}, \mathbf{d})$. Other parameters are $\tau=0.5 \mathrm{ps}$ and $T=300 \mathrm{~K}$.

\section{GCNW Waveguides}

In this section, we review the GCNW waveguides, and Figure 2 gives the cross-sections of various GCNW-based structures. We classify these structures into several groups: (a) and (b) for graphene-coated single circular/elliptical nanowire, (c) and (d) for coaxial-like GCNWs, (e) and (f) for GCNW pairs, (g-j) for GCNW-based hybrid waveguides, and (k-m) for GCNW 1D/2D arrays and trimer. For convenience, we have also listed the main parameters below to evaluate the waveguiding performances. 


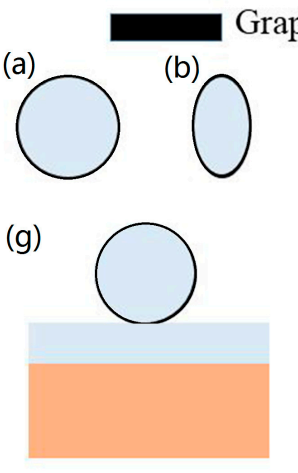

(k)

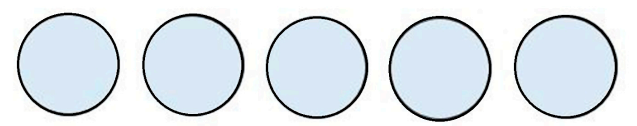

(d)

(c)<smiles>C1=CCCCCCC1</smiles>

(h)
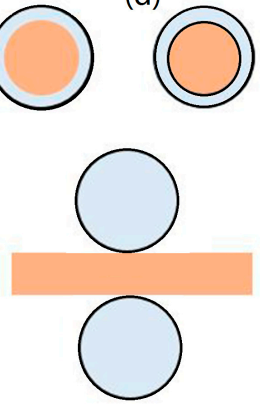

(i)<smiles>C1CCCCCCCCCCC1</smiles>

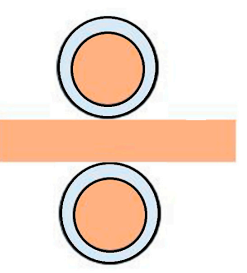

(l)

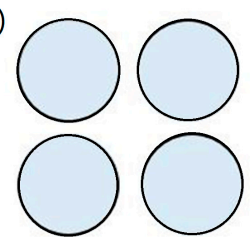

High-index dielectric

(f)<smiles>C1CC12CC2</smiles>

(j)

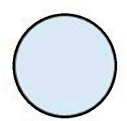

(m)

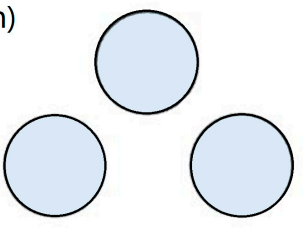

Figure 2. Various graphene-coated nanowire (GCNW)-based waveguides. (a) Circular and (b) Elliptical GCNWs. (c) Coaxial-like and (d) Long-range coaxial-like GCNWs. (e) Circular and (f) Bow-tie GCNW pairs. (g) Traditional and (h) symmetric GCNW-based hybrid waveguide. (i) Modified symmetric and (j) Triangle dielectric integrated GCNW-based hybrid waveguides. (k) 1D and (1) 2D arrays of GCNWs. (m) GCNW trimer.

Usually, propagation length $\left(L_{\mathrm{P}}\right)$, normalized mode area $\left(A_{\text {nor }}\right)$, and figure of merit $(\mathrm{FoM})$ are employed to quantitatively illustrate the waveguiding performances. The propagation length $L_{\mathrm{P}}$ is defined as the amplitude of the field decays to $1 / e$ of its initial value and calculated by $L_{\mathrm{P}}=\lambda_{0} /\left[2 \pi \operatorname{Im}\left(N_{\text {eff }}\right)\right]$, where $\lambda_{0}$ is the wavelength in vacuum and $\operatorname{Im}\left(N_{\text {eff }}\right)$ denotes the imaginary part of the effective mode index. The effective mode size $A_{\text {eff }}$ is evaluated by the ratio of the total mode energy and the maximum electromagnetic energy density [41]:

$$
A_{\mathrm{eff}}=\iint W(\mathrm{r}) d^{2} \mathrm{r} / \max [W(\mathrm{r})]
$$

where the electromagnetic energy density $W(r)$ is given by

$$
W(\mathrm{r})=\frac{1}{2}\left\{\frac{d[\varepsilon(\mathrm{r}) \omega]}{d \omega}|\mathrm{E}(\mathrm{r})|_{2}+\mu_{0}|\mathrm{H}(\mathrm{r})|^{2}\right\} .
$$

In Equation (7), $\mathrm{E}(\mathrm{r})$ and $\mathrm{H}(\mathrm{r})$ denote the electric and magnetic fields, respectively. $\varepsilon(\mathrm{r})$ is the electric permittivity and $\mu_{0}$ is the magnetic permeability in air. The normalized mode size is defined as $A_{\text {nor }}=A_{\text {eff }} / A_{0}$ with $A_{0}=\lambda_{0}^{2} / 4$ being the diffraction-limited mode size in air. Figure of merit (FoM) [42] is defined as the ratio of the mode propagation length to the diameter of the effective mode size, which is $\mathrm{FoM}=L_{\mathrm{p}} / \sqrt{A_{\mathrm{eff}} / \pi}$.

\subsection{Graphene-Coated Single Nanowires}

\subsubsection{GCNWs}

As far as we know, the study of the graphene-coated single nanowire (see Figure 2a) began with considering the photonic modes in $\mathrm{THz}$ region, where the graphene-coated semiconductor cylinder served as a photonic crystal [43]. Later, Zhao et al. [44] numerically studied the surface plasmon whispering gallery mode properties of the graphene-coated InGaAs nanowire cavity. A high cavity quality factor of 235 was obtained for a $5 \mathrm{~nm}$ radius cavity, along with a mode area (see Equation (6)) 
as small as $3.75 \times 10^{-5} \lambda_{0}^{2}$ (when normalized by $A_{0}=\lambda_{0}^{2} / 4$, the normalized mode area is $1.5 \times 10^{-4}$ ) at $\lambda_{0}=1.55 \mu \mathrm{m}$.

Important progress occurred in 2014. The eigen equation for the SP mode in the GCNW was achieved for the first time in THz [45] and mid-infrared bands [30]. It was found that the fundamental $\mathrm{TM}$ surface plasmon mode $\mathrm{TM}_{0}$ was cut-off free. In the work, Gao et al. [30] showed that with increasing nanowire permittivity, effective mode index almost linearly increased, which indicated a stronger localization of graphene plasmon mode, while larger modal loss (see Figure 3a). Also, high-order modes no longer existed at a small enough value of nanowire permittivity. A propagation length of $5.44 \mu \mathrm{m}$ (see Figure $3 \mathrm{~b}$ ) and a normalized mode area of $1.35 \times 10^{-3}$ could be obtained at $30 \mathrm{THz}$ with a radius of $100 \mathrm{~nm}$ and chemical potential fixed at $1 \mathrm{eV}$. Meanwhile, Gao et al. [31] presented an analytic modal cut-off wavelength formula, which could be applied to calculate the cut-off wavelength of each order mode easily and accurately. Results also indicate that the higher-order modes would be cut-off at certain chemical potential values, which means the cut-off frequencies of these modes depend on the inductive behavior provided by the graphene layer.

(a)

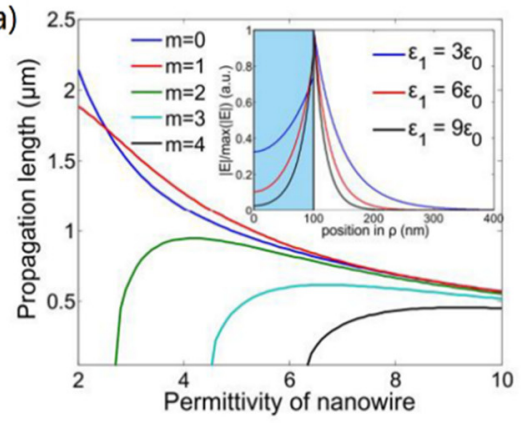

(b)

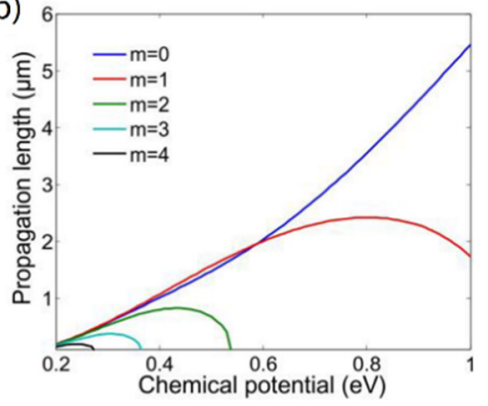

(c)
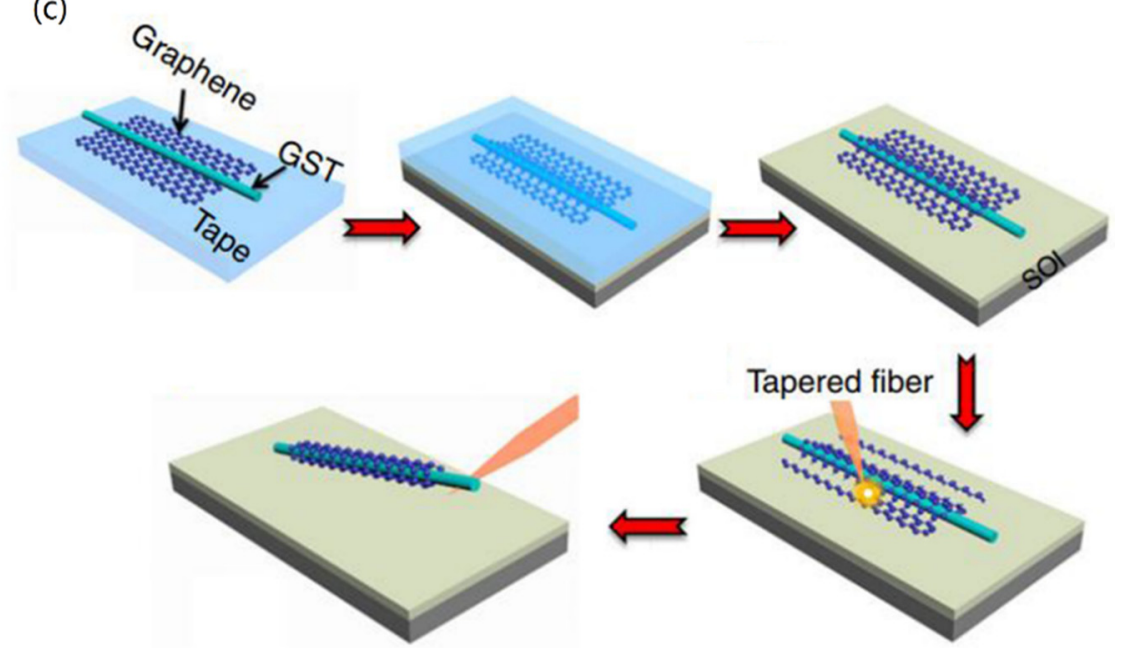

Figure 3. Propagation length as functions of nanowire permittivity (a) and chemical potential (b) at $30 \mathrm{THz}$ with $R=100 \mathrm{~nm}$. Reprinted with permission from reference [30]. Copyright Optical Society of America, 2014. (c) Schematic illustration of the GCNW fabrication process. Reprinted with permission from reference [46]. Copyright Springer Nature, 2018.

Apart from the theoretical analysis of GCNWs, several research teams reported [46-48] the fabrication of freestanding GCNWs for optical waveguiding, and Figure $3 \mathrm{c}$ is the schematic illustration of the GCNW fabrication process [46]. Also, He et al. [48] investigated the graphene-covered microfiber, and both theoretical and experimental results indicated that the proposed device can be used as a polarization dependent saturable absorber as well as an optical polarizer. Thus, they showed the dielectric nanowire can be easily coated by a graphene layer due to the van der Waals force. 
Owing to the strong optical field enhancement of graphene plasmons, Zhu et al. [49] proposed a graphene-coated tapered nanowire probe to achieve strong field enhancement in the mid-infrared frequencies. Based on the adiabatic approximation $\left|\mathrm{d}\left[\operatorname{Re}(\beta)^{-1}\right] / \mathrm{d} z\right|<<1[50]$, they analytically investigated the field enhancement along the tapered region, and results were consistent with the rigorous numerical simulations. Finally, the GCNW probe could achieve an order of magnitude larger field enhancement than the metal-coated probes, shown in Figure 4a. Later, they showed that a field enhancement as high as 24 could be achieved [51].

In 2016, Davoyan et al. [52] provided a performance comparison between the GCNW structure and some other typical $\mathrm{THz}$ waveguides. Results showed that the GCNW outperformed its metallic analog in modal field confinement, since surface plasmon modes in subwavelength metallic wire $\left(\sim \lambda_{0} / 50\right)$ radius were weakly confined at $\mathrm{THz}$ band. Later, Huang and Cong et al. [34,53] proposed the GCNWs with a drop-shaped cross-section for guiding THz plasmons, and an extremely long propagation length $(1 \mathrm{~mm})$ with a very small focal spot with full width at half maximum (FWHM) about $10 \mathrm{~nm}$ could be achieved, which resulted from the distinctive mode field distribution caused by both the top and bottom arcs of the waveguide. In 2019, Teng et al. [54] showed that graphene-coated elliptical nanowires (see Figure $4 \mathrm{~b}$ ) could be used for $\mathrm{THz}$ waveguiding, and a propagation length over $200 \mu \mathrm{m}$ as well as a normalized mode area of approximately $10^{-4} \sim 10^{-3}$ could be obtained at $3 \mathrm{THz}$. Increasing long-short axis ratio could simultaneously achieve both long propagation length and very small FWHM of the focal spots. For $b / a=10$, a pair of focal spots about $40 \mathrm{~nm}$ could be obtained. In mid-infrared band, the elliptical GCNW [55] was investigated in the elliptical cylinder coordinate system, and the dispersion equation was obtained by using the separation variable method with the Mathieu functions. A propagation length around $2 \mu \mathrm{m}$ could be obtained when $a=110 \mathrm{~nm}$, $b=80 \mathrm{~nm}, \mu_{\mathrm{c}}=0.72 \mathrm{eV}$, and $\lambda_{0}=7 \mu \mathrm{m}$. The long and short axes of the elliptical nanowire have a significant influence on the mode properties. A few months before, Wu et al. [56] studied the dispersion equation of a GCNW, and found there was another branch of guided modes, called photonic-like modes. The propagation distances of these photonic-like modes could be five orders of magnitude longer than those of the SP modes.

(a)
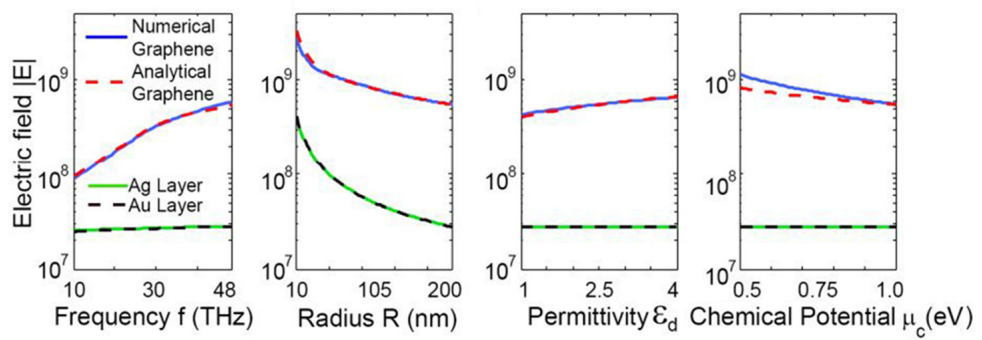

(1)
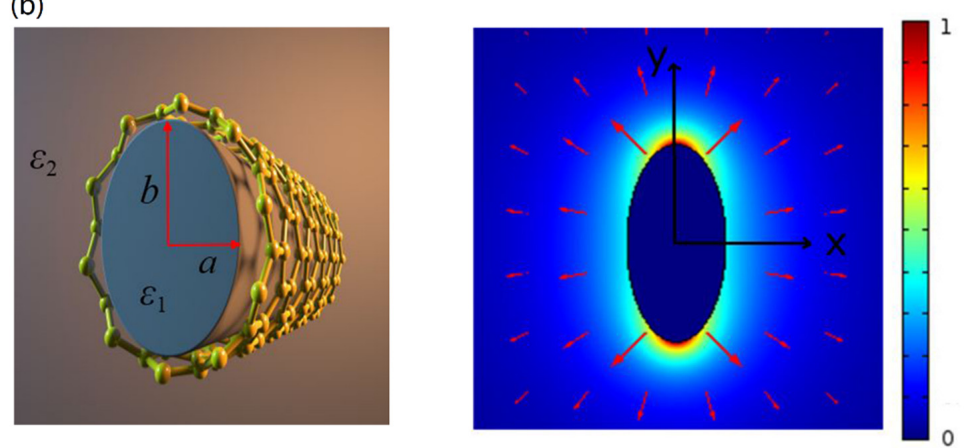

Figure 4. (a) The electric fields $|\mathrm{E}|$ of the graphene-coated and metal-coated nanowire waveguides as functions of frequency, nanowire radius $R$, nanowire permittivity, and chemical potential. Reprinted with permission from reference [49]. Copyright Optical Society of America, 2014. (b) Graphene-coated elliptical nanowire and normalized electric field distribution [54]. 
It is worth mentioning that localized surface plasmon mode [57] and TE plasmon mode [58] in GCNWs have also been investigated. The report showed that the negativity of graphene conductivity's imaginary part was not a sufficient condition, and the GCNW supported TE plasmon mode when the core radius of waveguide was larger than the critical value. Results also indicated that the critical radius depended on the frequency and the index-contrast between the inside and outside materials of the waveguide.

\subsubsection{Coaxial-like GCNWs}

In 2016, Liu et al. [35] proposed another kind of GCNW waveguides, and we call them coaxial-like GCNW, shown in Figure 2c. The coaxial-like GCNW is composed of a silicon nanowire core surrounded by a silica layer and then a graphene layer, and it is also similar to the cylindrical hybrid plasmonic waveguide, which is composed of a low-index dielectric layer sandwiched between a high-index dielectric layer and a graphene layer (or metal). Based on analytical study and numerical simulation, an ultra-small normalized mode area about $\sim 10^{-5}$ and a large propagation length about $8 \mu \mathrm{m}$ can be achieved at the wavelength of $7 \mu \mathrm{m}$, which outperformed the graphene-coated single nanowires. Then, Liu et al. [59] proposed a coaxial-like GCNW long-range waveguide (see Figure 2d), in which an extra graphene layer on the silicon nanowire core was added. The symmetric coupling and anti-symmetric coupling mode originated from the constructive interference and destructive interference of the two GCNWs were intensively investigated. For the fundamental long-range $\left(L_{0}\right)$ mode, when $\lambda_{0}=10 \mu \mathrm{m}$, $\mu_{\mathrm{c}}=0.6 \mathrm{eV}, R=24 \mathrm{~nm}$, and $t=12 \mathrm{~nm}, L_{\mathrm{P}}$ is about $9 \mu \mathrm{m}$, while the normalized mode area is still $\sim 10^{-5}$. Further, the propagation length could be increased with increasing $\mu_{\mathrm{c}}$.

Recently, Zhao et al. [60] theoretically investigated the plasmon modes in a circular cylindrical double-layer graphene structure, which was similar to coaxial-like GCNW long-range waveguide. By solving the Maxwell equations together with boundary conditions, they obtained the electromagnetic fields in each region and then the dispersion equation. Particularly, results showed that the trade-off between mode confinement and propagation loss was broken at large inner graphene layer to outer graphene layer distances. As a consequence, both strong mode confinement and longer propagation length can be achieved. Also, the modal property of an elliptical coaxial-like GCNW [61] was analyzed by using the separation of variables method. The surface conductivity of graphene was modulated by employing a DC bias, which was highly important to study the tunable properties of the graphene-based plasmonic devices. Serrano et al. [62] investigated the propagation of surface plasmon modes along three kinds of electrically and magnetically biased GCNWs in THz band, and designed a plasmonic dipole antenna.

\subsection{GCNW Pairs}

The GCNWs have shown excellent optical performances, such as low propagation loss and fundamental mode cut-off free. However, the $\mathrm{TM}_{0}$ mode with a radially polarized electric field (with field components of $E_{r}, H_{\phi}, E_{z}$ ) in a GCNW remains weakly localized, since the field exponentially decays away from the interface. This problem is later addressed by using a GCNW pair, shown in Figure 2e,f, which is an analogy of metal two-wire waveguides [63,64].

The study of GCNW pairs began with investigating the field enhancement and gradient force [65]. Then Zhai et al. [66] studied a GCNW pair with elliptical cross-section by using the finite element method, and found the modal properties can be adjusted finely by the elliptical semiminor axis. Peng et al. [67] analyzed the mode properties of an asymmetric GCNW pair waveguide by the multipole expansion method. These reports laid emphasis either on the field enhancement or effective mode index of the fundamental plasmon mode, while lacking a comprehensive evaluation of the waveguiding performances of GCNW pairs. In 2019, Teng et al. [33] fully investigated the waveguiding performance of the GCNW pairs in the mid-infrared range. Results indicated that the fundamental quasi-TM plasmon mode could achieve a propagation length about $9 \mu \mathrm{m}$, while the normalized mode area is only $10^{-4}$, which was one order of magnitude smaller compared with the GCNWs. 
Further investigations showed reducing nanowire permittivity could decrease the modal loss. Due to the circular geometry, the field was still weakly confined. Based on the bow-tie shaped metallic structures, a bow-tie type GCNW pair [68] was proposed, shown in Figure 2f. Benefiting from the sharp tip, the normalized mode area approached to an order of $10^{-7}$ magnitude, which was greatly reduced compared with other reports. Later, Teng et al. [69] theoretically showed that high-performance and low-loss transmission of graphene plasmons can be achieved by adding a silica substrate to the GCNW pairs, shown in Figure 5. Furthermore, the results showed that inserting a low index material layer between the nanowire and substrate could compensate for the loss accompanied by the substrate. Recently, Raad et al. [70] proposed a 3D graphene-coated nano-disk dimers to achieve multi-frequency near-field enhancement, which originated from the excitation of hybridized localized surface plasmons on top and bottom faces of the disks along with the mutual coupling from the adjacent particle.

(a)

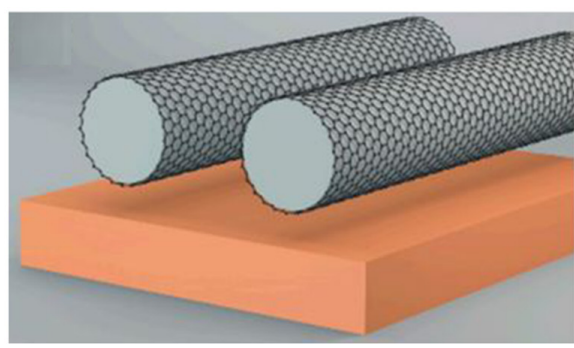

(b)

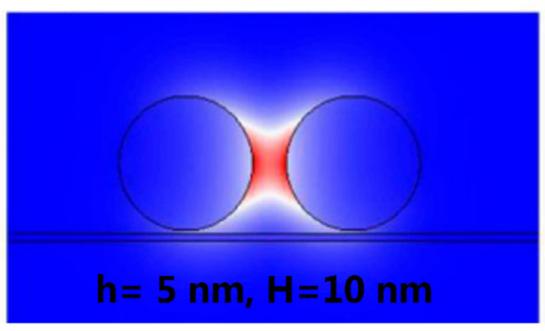

Figure 5. (a) Schematic of GCNW pair above a substrate, and (b) normalized electric field distribution of the fundamental graphene plasmon mode at $30 \mathrm{THz}$ [69].

The GCNW pairs show distinct advantage over the GCNWs. However, the trade-off between modal loss and field confinement still exists in the GCNW pairs, which hinders the practical applications of these configurations in integrated photonics. Despite the recent achievements, further reducing the modal field size while increasing (or maintaining) the transmission distance remains a huge challenge. On the other hand, in most previous studies, GCNWs and GCNW pairs are assumed to be surrounded by air, which means that the GCNWs are suspended without support. For applications in photonic integration circuits [71], a buffer or substrate is indispensable. Next, we will show how to address this obstacle.

\subsection{GCNW-Based Hybrid Waveguides}

To further enhance the optical performances of the GCNWs, it was proposed that when the GCNW was located adjacent to a high-index dielectric substrate, a substrate-supported GCNW with extreme small modal field can be realized. Meanwhile, a huge reduction of the modal propagation distance emerges due to the optical energy leakage into the substrate, which causes a great challenge for the implementation of long-range optical energy transmission.

Inspired by the metallic hybrid plasmon waveguide [41], which consists of a dielectric nanowire separated from a metal surface by a nanoscale dielectric gap. Hajati et al. [72] theoretically showed that high-performance and low-loss mid-infrared plasmon waveguiding could be achieved by inserting a thin low-index dielectric layer between a GCNW and a high-index substrate, shown in Figure $2 \mathrm{~g}$. It is well known that this hybrid mode originated from the coupling of graphene plasmon mode and the photonic mode inside the high-index substrate, which is similar to metal hybrid plasmon waveguide [41]. Therefore, the GCNW-based hybrid waveguides offer a better compromise between the loss and confinement than GCNWs. In 2017, Hajati et al. [73] proposed a symmetric GCNW-based hybrid waveguide, which comprised two vertically coupled GCNWs integrated with a thin high-index dielectric substrate, shown in Figure $2 \mathrm{~h}$. Through optimal design, a surface plasmon mode with high optical performance and low propagation loss can be achieved in the proposed structure. Results also showed a highly improved FoM with nearly two-fold electric field enhancement can be achieved 
compared with the plasmon mode in a GCNW over substrate. The modal propagation length could exceed $10 \mu \mathrm{m}$, and the normalized mode area is only $10^{-5}$ (see Equation (6)).

Later, $\mathrm{Wu}$ et al. [74] further reduced the normalized mode size down to only $10^{-7}$ by using a GCNW-based hybrid waveguide integrated with triangle wedge substrate and the low-index dielectric gap, shown in Figure $2 \mathrm{j}$. At the same time, the graphene plasmon mode could propagate several micrometers. Also, a modified symmetric GCNW-based hybrid waveguide [75] consisting of two vertically coupled double-graphene-coated nanowires integrated with a thin high-index dielectric substrate was investigated (see Figure 2i). Results showed under certain conditions, the proposed waveguide (Figure 2i, Type B) has better mode performance (FoM) over the symmetric GCNW-based hybrid waveguide (Figure 2h, Type A), shown in Figure 6. A novel hybrid plasmonic waveguide [76] based on the graphene-coated V-groove and the GCNWs was also proposed.
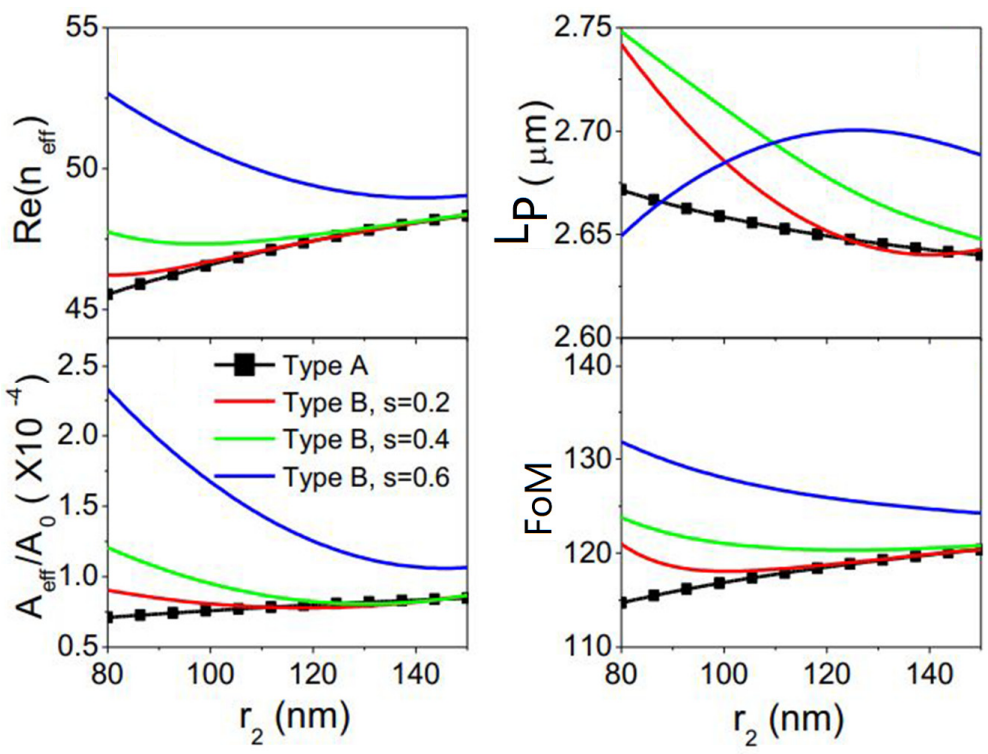

Figure 6. Comparison of the performance of two kinds of waveguides illustrated in Figure 2h,i. Reprinted with permission from reference [75]. Copyright Elsevier, 2019.

In the literature, a lot of promising graphene-based hybrid waveguides [77-86] were analyzed in $\mathrm{THz}$ and mid-infrared bands. Here, we mainly focus on the GCNWs and GCNW-based hybrid waveguide, thus they will not be covered here.

\subsection{GCNW Arrays}

Recently, graphene plasmonic solitons were predicted in graphene planar sheets and ribbons [87]. Also, researchers found that discrete plasmonic soliton modes could exist in 1D and 2D arrays of GCNWs [88], shown in Figure 2k,l. The waveguides coupling, discrete diffraction, as well as nonlinear modes were investigated by strictly solving the Maxwell's equations. Results showed the soliton propagation length could over $10 \mu \mathrm{m}$, shown in Figure $7 \mathrm{~b}$. It can be seen that the real part of the diffraction curve minimized at the Brillouin zone center $\left(k_{\mathrm{x}}=0\right)$ (see Figure $7 \mathrm{c}, \mathrm{d}$ ), implying a negative coupling as in other types of plasmonic waveguides. The increased band curvature at $\mu_{\mathrm{c}}=1.1 \mathrm{eV}$ reflected the stronger discrete diffraction. Meanwhile, the imaginary part, which reflected the propagation loss of the Bloch modes, arrived at its minimum at the edge of the Brillouin zone $\left(k_{\mathrm{x}}=\pi / \mathrm{s}\right)$, and significantly decreased as the coupling became stronger (at larger $\mu_{\mathrm{c}}$ ). Later, Meng et al. [89] investigate the Bloch and topological edge plasmon modes in a 2D GCNW arrays. Due to the strong confinement of graphene plasmons, the modal wavelength of topological edge modes can be only $\lambda_{0} / 20$. Each gap possessed two degenerate topological edge modes. The imaginary parts of effective 
mode indices of the topological edge modes are 0.378 and 0.42 , and the corresponding propagation distances are $2.53 \mu \mathrm{m}$ and $2.27 \mu \mathrm{m}$, respectively.

(a)

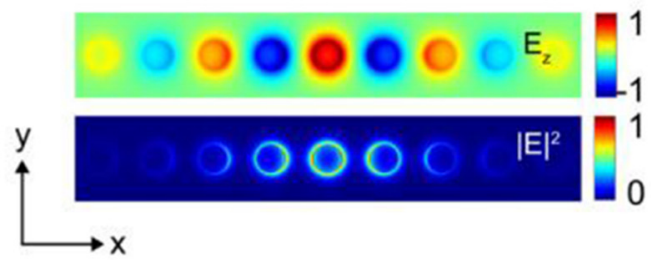

(c)

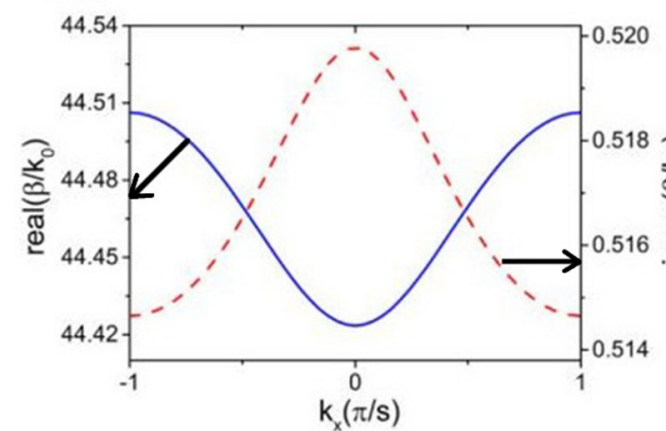

(b)

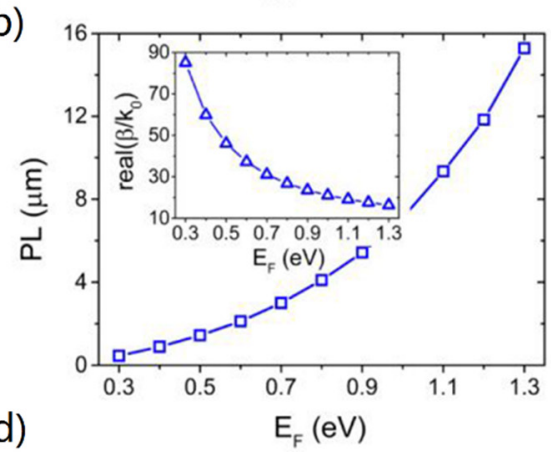

(d)

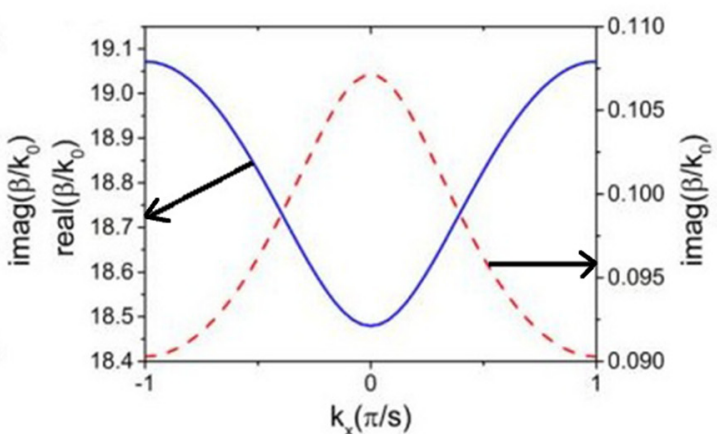

Figure 7. (a) Field distribution of a 1D GCNW array. (b) The soliton propagation length, insert for soliton mode index. Diffraction relation of the fundamental transmission band, $\mu_{\mathrm{c}}=0.5 \mathrm{eV}$ for (c) and $1.1 \mathrm{eV}$ for $(\mathbf{d}) . \lambda_{0}=10 \mu \mathrm{m}, a=100 \mathrm{~nm}$, and $s=4 a$. Reprinted with permission from reference [88]. Copyright Optical Society of America, 2016.

Recently, the trimers of GCNWs (see Figure 2m) with a non-coplanar [90] and coplanar [91] axis were also proposed to guide mid-infrared and terahertz waves, and the lowest modal fields were shown in Figure $8 \mathrm{a}, \mathrm{b}$. These two structures were analyzed to obtain the effective mode index by using the multipole method. The first five modes were systematically investigated in terms of field distributions and propagation properties, and the analytical results were consistent with the simulations.

(a)

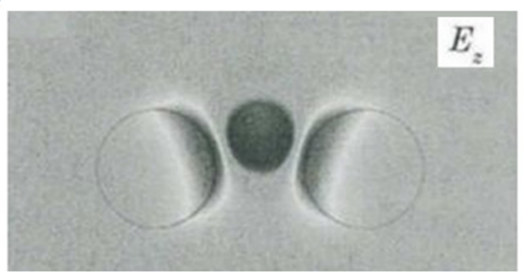

(b)

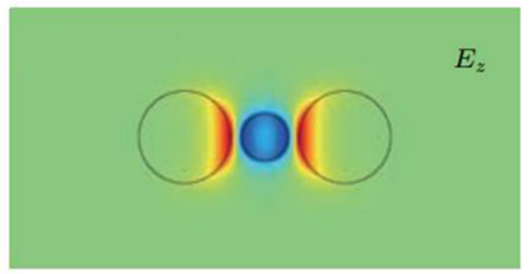

Figure 8. Field distributions $\left(E_{\mathrm{Z}}\right)$ of the lowest-order mode in non-coplanar (a) and coplanar (b) GCNW trimers. (a) Reprinted with permission from reference [90]. Copyright Chinese Laser Press, 2019. (b) Reprinted with permission from reference [91]. Copyright Chinese Physical Society, 2018. 
Apart from the study of the modal propagation properties, and the excitation of plasmon modes in the GCNWs was also significant. Usually, surface plasmon modes could be excited by using periodic structures such as metal gratings [92,93]. Several approaches [94-98] have been proposed to excite graphene plasmons in graphene planar structures. In 2016, Xia et al. [99] demonstrated an effective solution to excite localized surface plasmons on GCNW arrays, and the excited localized mode was compared with that of graphene nanoribbons. The excited resonance frequency showed an obviously blue shift, along with the narrowing of FWHM, which was due to low occupation ratio and smaller width of the incident light in rolled graphene, thus only the shorter wavevector can couple into the plasmon wave.

\section{Applications of GCNWs}

As indicated before, plasmon modes in GCNWs offer some important advantages, for example, tunability by changing the surface conductivity, extremely strong modal field localization, and huge field enhancement. These unprecedented properties make a good promotion of various applications in many fields. Here, we briefly review some of the major applications of GCNWs.

\subsection{Photonic Devices}

The applications of GCNW-based tunable nanoscale devices are very important in photonics integration. Based on graphene cylindrical resonators, Asgari et al. [100] proposed a refractive index sensor, a power splitter, and a four-channel multi/demultiplexer. The proposed structure was composed of two or four graphene sheets as its input/output ports and a graphene cylindrical resonator. Cao et al. $[46,101]$ proposed a chiroptical switch based on chiral plasmons in a graphene-coated $\mathrm{Ge}_{2} \mathrm{Sb}_{2} \mathrm{Te}_{5}$ (GST225) nanowire. Results showed that the chiral SPs propagating along the nanowire can be reversibly switched between "on" (transparent) and "off" (opaque) as transiting the state of GST225 core between amorphous and crystalline, shown in Figure 9a,b. And the short phase transition times of $2.4 \mathrm{~ns}$ and $8 \mathrm{~ns}$ can be obtained, resulting in a fast switching on/off. Gan et al. [102] proposed an all-fiber phase shifter based on graphene's strong optical absorption and excellent thermal properties, and the proposed graphene-coated microfiber enabled all-optical switching with an extinction ratio of $20 \mathrm{~dB}$ and a rise (fall) time of $9.1 \mathrm{~ms}(3.2 \mathrm{~ms})$.

Optical isolators [103] are crucial for nanoscale photonic applications. Pae et al. [104] demonstrated a graphene waveguide ring resonator, consisting of a GCNW and a graphene layer, allowed a nanoscale platform for a high-contrast optical isolator. The magnetically controlled graphene structure for nanoscale high-contrast optical isolator was due to plasmon resonance enhancement combined with resonator resonance enhancement.

Polarization behavior has a profound impact on the performance of optical devices. The control of polarization in the GCNW was reported in Reference [48]. It was reported that the saturated absorption could be achieved by using a graphene layer covered on the upper surface of the microfiber. Particularly, when the microfiber radius reduced to $1 \mu \mathrm{m}$, such graphene-microfiber hybrid waveguide can be utilized as TM polarizer, which implied that the graphene-coated microfiber could be used for optical communication. Subsequently, Kou et al. [105] demonstrated polarization manipulation by a GCNW. The ultra-long light-graphene interaction was implemented by a graphene-integrated helical microfiber device, shown in Figure 9 c. The proposed device can operate as not only a broadband polarizer but also a high-Q (nearly $2 \times 10^{4}$ ) single-polarization resonator. By employing a two-coil structure, an extinction ratio as high as $\sim 16 \mathrm{~dB}$ was obtained over a $450 \mathrm{~nm}$ bandwidth. 
(a)

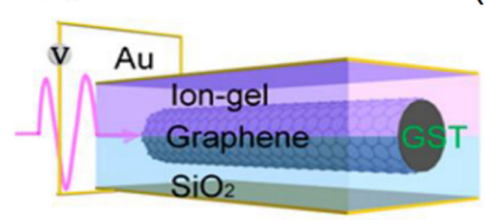

(b)

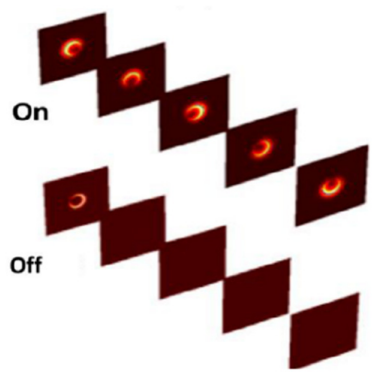

(c)

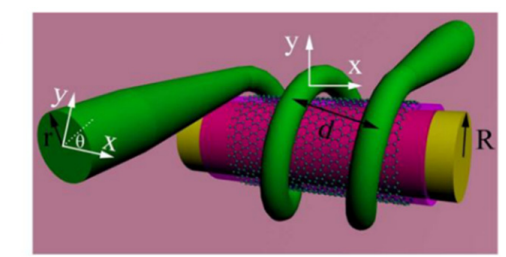

Figure 9. (a) Sketch of the graphene-coated GST225 nanowire and (b) switching "on/off" chiral SP modes at $\mu_{\mathrm{c}}=0.6 \mathrm{eV}$. Reprinted with permission from reference [101]. Copyright American Chemical Society, 2018. (c) Schematic of a graphene-based nanowire in-line polarizer with a two-coil structure. Reprinted with permission from reference [105]. Copyright Optical Society of America, 2014.

\subsection{Ultrafast Optical Modulators}

Electro-optic modulators are among the most important components for optical communication. For photonic integration, optical modulators with high modulation speed, small size, and large bandwidth are preferred.

In 2011, Liu et al. [106] demonstrated a high-speed graphene-based optical modulator. By electrically tuning the Fermi level of a graphene film, a broad operation spectrum and very small device area of merely $25 \mu \mathrm{m}^{2}$ were achieved. Later in 2014, Li et al. [107] reported that a graphene-coated microfiber all optical modulator could achieve a modulation depth of $38 \%$ and a response time of $\sim 2.2$ ps. Also, an in-line, all-optical fiber modulator based on a stereo graphene-coated microfiber structure was demonstrated [108], shown in Figure 10a. The all-optical modulation mechanism was based on the Pauli blocking effect. Results showed a modulation depth of $7.5 \mathrm{~dB}$ and a modulation efficiency of $0.2 \mathrm{~dB} / \mathrm{mW}$ could be achieved.

(a)

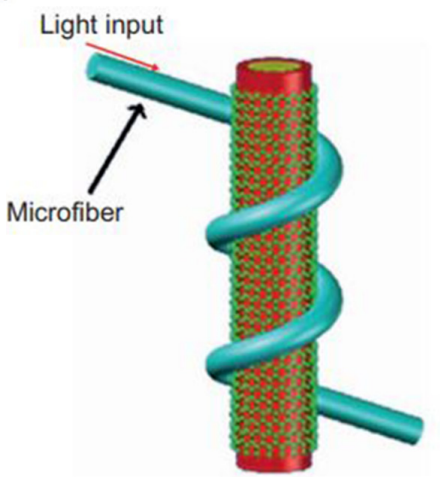

(b)

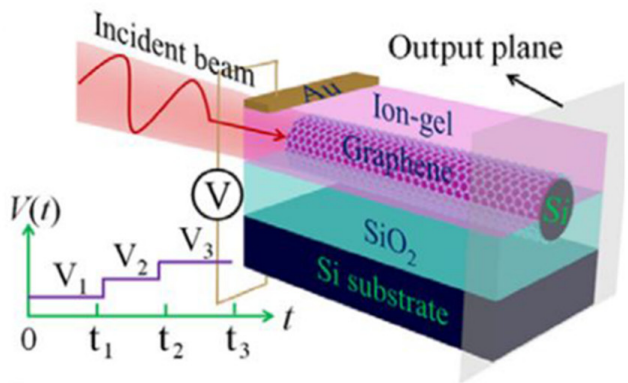

Figure 10. (a) A stereo graphene-coated-microfiber modulator. Reprinted with permission from reference [108]. Copyright Springer Nature, 2015. (b) Gate-programmable electro-optic addressing scheme. Reprinted with permission from reference [109]. Copyright American Chemical Society, 2016.

Recently, Liang et al. [109] derived the eigenmode equations of both tightly confined EH (Plasmon modes dominated by a longitudinal electric field) and TM SP modes supported by the GCNW and analytically and numerically studied their modal characteristics. Significantly, both the period of the 
swing beam and the chirality and period of the helix could be modulated by tuning the applied gate voltage on graphene. The proposed nanowire system, shown in Figure 10b, offered a way for nanoscale photonic devices at sub-10 nm scale.

\subsection{Nonlinear Optics}

Graphene plasmonic waveguides offer huge field intensity on the surface of graphene, which will enhance nonlinear effects significantly. We outline the main applications of GCNW in this field.

\subsubsection{Saturable Absorber in Fiber Lasers}

In this subsection, we show that the GCNWs could work as a saturable absorber in fiber lasers. In 2012, He et al. [110] reported that based on the reduced graphene oxide (RGO) deposited on the surface of the microfiber by use of high-temperature heating, a direct generation of doublet ultra-wide-band pulses were observed by using of a passively mode-locked fiber laser. The strong interaction of the RGO with the evanescent field of the microfiber in the fiber laser system caused the saturable absorption effect. Also, results indicated the ultra-wide-band doublet pulses could be directly generated through the interaction between the dispersion and nonlinearity in the laser cavity. However, the modulation depth was only 5.75\%. In 2014, the same team investigated a polarization-dependent saturable absorber [48] based on a graphene layer covered on the upper surface of the microfiber. Through reducing the radius of microfiber, the strong light-graphene interaction occurred via the evanescent field of the guided mode in the microfiber. When the radius of the microfiber is $0.8 \mu \mathrm{m}$, its polarization extinction ratio is $\sim 27 \mathrm{~dB}$. When the radius of microfiber reached $\sim 3 \mu \mathrm{m}$, a polarization-dependent saturable absorber could be obtained with high thermal damage threshold of $\sim 975.82 \mathrm{MWcm}^{-2}$ for p-polarization and $\sim 1233.2 \mathrm{MWcm}^{-2}$ for s-polarization, and its polarization-dependent modulation depth varied from $\sim 10.25 \%$ to $\sim 12.85 \%$. In the same year, Zhao et al. [111] reported on the generation of dual-wavelength rectangular pulses in a Yb-doped fiber laser by using a graphene-coated microfiber saturable absorber.

Later, Liu et al. [112] exploited a graphene-coated microfiber saturable absorber (see Figure 11) in a mode-locked fiber laser for the generation of ultrafast pulses. The proposed all-surface technique can realize a higher efficiency of light-graphene interactions, and the GCNW-based saturable absorber could generate ultrafast optical pulses within $1.5 \mu \mathrm{m}$. Yao et al. [113] demonstrated a compact all-in-line graphene-based distributed feedback Bragg-grating fiber laser with narrow linewidth based on GCNWs. In 2018, Wang et al. [114] reported that using a graphene-coated microfiber saturable absorber, the generation and evolution of multiple operation states were proposed and demonstrated in passively mode-locked thulium-doped fiber laser. Recently, Li et al. [115] reported an active-passive Q-switched laser based on graphene-covered microfiber, which not only served as a passive saturable absorber in a single laser cavity, but also could be used as an all-optical modulation device.

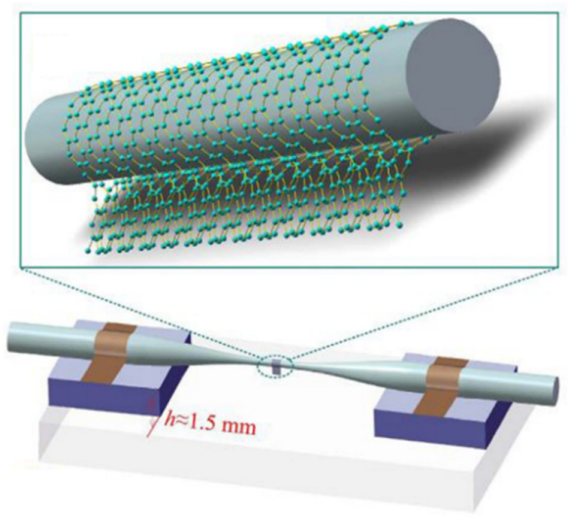

Figure 11. The GCNW saturable absorber. Reprinted with permission from reference [112]. Copyright Springer Nature, 2016. 


\subsubsection{Optical Bistability}

Optical bistability [116] is a rapidly expanding field of current research because of its potential application to all-optical logics and switching. However, in nonlinear plasmonics, the switching threshold of optical bistability is limited by the weak nonlinear responses from the conventional Kerr dielectric media. To enhance the nonlinear responses, GCNWs were employed to beat this limitation. Based on GCNWs, Li et al. [117] developed a nonlinear scattering model under the mean field approximation and studied the bistable scattering in a GCNW based on the semi-analytical solutions. It was found that the switching intensities of bistable scattering can be smaller than $1 \mathrm{MW} / \mathrm{cm}^{2}$ at the working frequency. Meanwhile, another team [118] studied the optical bistability of GCNWs with Kerr-type nonlinear response within the framework of both nonlinear full-wave scattering theory and nonlinear quasi-static theory. Typical optical bistable properties were observed, and results indicated when high electromagnetic field was applied, nonlinear full wave theory yielded a new bistable region, indicating the existence of an artificial tunable magnetic dipole. These results could offer guidance to application of optical bistability in the high-speed all-optical communication.

\subsubsection{Other Nonlinear Effects}

Thanks to the huge field enhancement of graphene plasmons in GCNWs, lots of other nonlinear effects were investigated, such as four waves mixing [119], second harmonic generation [120], nonlinear plasmon coupling [121], magneto-optical Faraday effect [32,122], etc.

\subsection{Optical Cloaking}

Artificial metamaterials can bend light in almost any manner, and could be used to manipulate electromagnetic waves to achieve optical cloaking based on transformation optics [123-125].

Due to the tunability of surface conductivity of graphene, GCNWs were used to develop tunable invisibility cloaks. For the first time, drastically reduced overall visibility of the scattering object that was conducted via a GCNW in the THz region (see Figure 12a,b) by Chen and Alu [126]. Recently, Naserpour et al. [127] investigated, both theoretically and numerically, a graphene-coated nano-cylinder illuminated by a plane wave. Results showed that the polarization-dependent effect leaded to tunable resonant invisibility that can be achieved via modification of graphene chemical potential. The scattering efficiency and field distributions were shown in Figure 12c,d. Later, invisibility in a trimer of GCNWs was presented by Fesenko et al. [128]. For TM-polarized incident waves, the normalized scattering cross-section spectra of all structures (GCNW, GCNW dimer, GCNM trimer) behaved similarly and exhibited a single invisible region. However, in the case of the TE-polarized incident wave, the normalized scattering cross-section spectra of the GCNW and both dimer and symmetric trimer clusters differed noticeably from each other. The strong coupling between plasmon modes of individual nanowires gave rise to several plasmonic resonances and invisibility regions in the scattering spectra. In 2019, dual-polarized all-angle cloaking was demonstrated [129] by using a helical graphene ribbon coated nanowire, shown in Figure 13a,b. It has been shown that the frequency can be widely tuned with the pitch angle, period, and width of graphene strips, and the most optimal frequency of all-angle cloaking is $13.15 \mathrm{THz}$. 


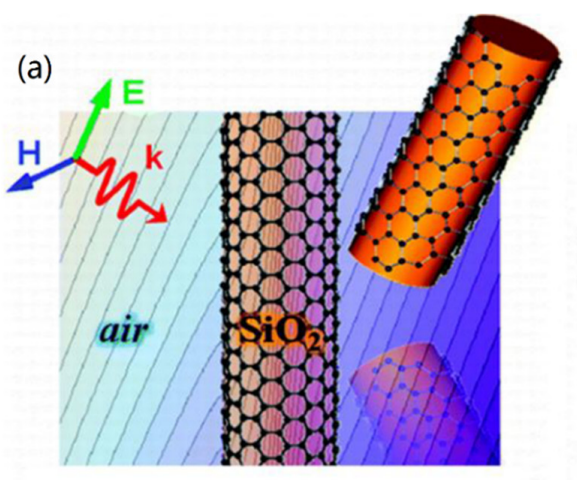

(b)

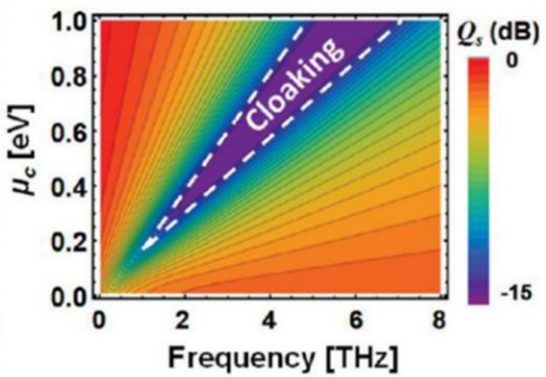

(c)

(d)
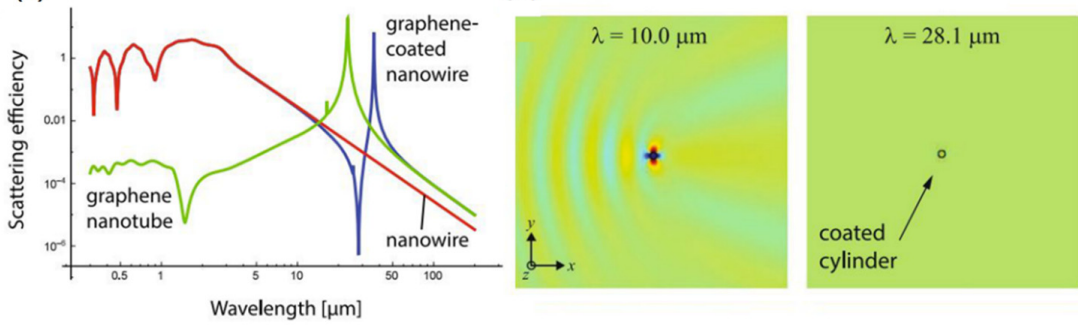

Figure 12. (a) Graphene-coated cylindrical object. (b) Scattering gain $\left(Q_{s}\right)$ for the graphene cloak varying chemical potential and the frequency of operation with $\tau=0.5 \mathrm{ps}$. Reprinted with permission from reference [126]. Copyright American Chemical Society, 2011. (c) Scattering efficiencies $\left(Q_{\text {sca }}\right)$ of three structures. (d) Normalized electric field $\left|\mathrm{E} / \mathrm{E}_{0}\right|$ for a GCNW with $R=0.5 \mu \mathrm{m}$ illuminated by a transverse-electric (TE)-polarized plane wave at $\lambda_{0}=10.0 \mu \mathrm{m}$ and $28.1 \mu \mathrm{m}$, corresponding to the invisibility window in (c). Reprinted with permission from reference [127]. Copyright Springer Nature, 2017.

(a)

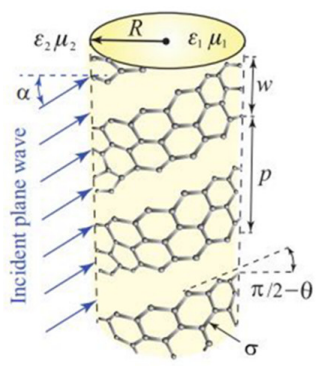

(b)

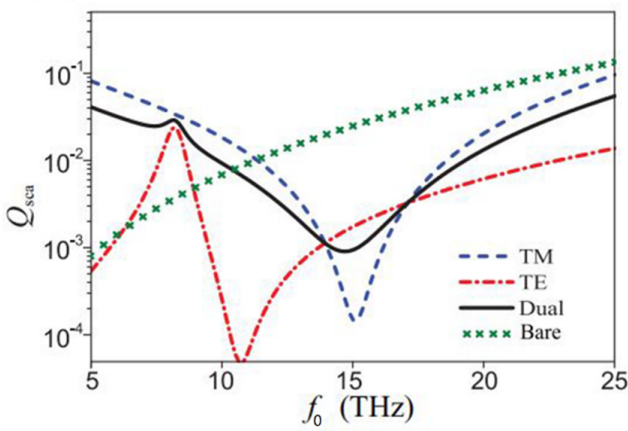

Figure 13. (a) Dielectric cylinder coated by helical graphene strips for oblique wave incidence. (b) Scattering efficiency versus wave frequency for a GCNW under normal incidence of TE, transverse-magnetic (TM), and dual-polarized waves. Reprinted with permission from reference [129]. Copyright American Physical Society, 2019.

Furthermore, a modified transformation optics approach was employed to study the plasmonic interactions between two GCNWs [130]. It was found that the interaction between two GCNWs resulted in polarization-independent multi-frequency Fano dips, which showed a broadband red shift of bonding modes and a blue shift of anti-bonding modes when the nanowires approached each other.

\subsection{Other Applications}

Although we have summarized some of the major areas of the GCNW research, the list is far from complete. Lots of other intriguing applications have been demonstrated. For instance, owing to the strong field enhancement of graphene plasmons, Zhu et al. [65] investigated the optical force, 
which was more than one order of magnitude larger than silver nanowire pairs, in the slot region of the GCNW dimer. Yang et al. [131] studied the optical forces exerted on a graphene-coated dielectric particle by a focused Gaussian beam.

Plasmon-induced-transparency and slow light effect were studied [132] in a GCNW system consisting of two identical-shaped GCNWs placed at either side of the graphene waveguide. Tunable Fano resonance [133] was observed in a system comprised of a point-like emitter near a GCNW. Results showed the Fano line shape of transition rates can be tailored and electrically tuned by varying the distance between emitter and cylinder and by modulating the graphene chemical potential. Recently, a report showed [134] that enhanced electromagnetic energy transfer between the donor and acceptor quantum emitters could be fulfilled by using GCNW surface plasmons.

\section{Conclusions and Perspective}

Although the GCNW is an emerging research field, the achievements in this field have been truly impressive. A lot of promising devices, including waveguides, polarizers, modulators, lasers, nonlinear devices, etc., have been proposed and experimentally demonstrated. However, the applications of GCNWs in other areas where the GCNW can fulfill its potential remain relatively unexplored. For instance, a combination of GCNWs with conventional plasmonic nanostructures. As literature has shown, many GCNW-based subwavelength waveguides have been theoretically investigated, however, there is a lack of experimental work in this area. We also note that the current reports mainly focus on mid- and near-infrared bands, and the application of GCNWs in THz band is relatively less studied. Although graphene plasmons show very strong field localization, due to the high absorption of the graphene layer, the modal propagation length of GCNW-based waveguide is limited. Therefore, further reducing the Ohmic loss is still a huge challenge. Finally, considering the attractive features of strong light-matter interactions, giant field enhancement, and tunability of graphene plasmons, we foresee GCNWs becoming the platform of choice for applications in the fields of the subwavelength photonic devices, super-resolution imaging, enhancing optical force, and nonlinear optics.

Author Contributions: Conceptualization, D.T.; writing-original draft preparation, D.T.; writing-review and editing, K.W. and Z.L.; visualization, K.W.; supervision, D.T. and K.W. All authors have read and agreed to the published version of the manuscript.

Funding: This work was supported by the Open Research Fund of Zhengzhou Normal University, the Scientific Research Starting Foundation of Zhengzhou Normal University.

Acknowledgments: The authors would like to acknowledge Minning Zhu from Rutgers, the State University of New Jersey for useful discussions.

Conflicts of Interest: The authors declare no conflict of interest.

\section{Abbreviations}

$\begin{array}{ll}\text { GCNWs } & \text { Graphene-coated nanowires } \\ \text { THz } & \text { Terahertz } \\ \text { SPs } & \text { Surface plasmons } \\ 1 / 2 / 3 \mathrm{D} & \text { One/two/three-dimensional } \\ \mathrm{TM} & \text { Transverse-magnetic } \\ \mathrm{TE} & \text { Transverse-electric } \\ \text { FWHM } & \text { Full width at half maximum } \\ \text { RGO } & \text { Reduced graphene oxide } \\ N_{\mathrm{eff}} & \text { Effective mode index } \\ f_{0} & \text { Frequency } \\ \lambda_{0} & \text { Wavelength in vacuum } \\ \mu_{\mathrm{c}} & \text { Chemical potential of graphene } \\ L_{\mathrm{P}} & \text { Propagation length } \\ \mathrm{R} & \text { Radius of dielectric nanowire } \\ \varepsilon_{\mathrm{d}} & \text { Permittivity of dielectric nanowire }\end{array}$




\section{References}

1. Barnes, W.L.; Dereux, A.; Ebbesen, T.W. Surface plasmon subwavelength optics. Nature 2003, 424, 824-830. [CrossRef]

2. Ozbay, E. Plasmonics: Merging photonics and electronics at nanoscale dimensions. Science 2006, 311, $189-193$. [CrossRef] [PubMed]

3. Zayats, A.V.; Smolyaninov, I.I.; Maradudin, A.A. Nano-optics of surface plasmon polaritons. Phys. Rep. 2005, 408, 131-314. [CrossRef]

4. Gramotnev, D.K.; Bozhevolnyi, S.I. Plasmonics beyond the diffraction limit. Nat. Photonics 2010, 4, 83-91. [CrossRef]

5. Sorger, V.J.; Oulton, R.F.; Ma, R.M.; Zhang, X. Toward integrated plasmonic circuits. MRS Bull. 2012, 37, 728-738. [CrossRef]

6. Kim, J.T.; Choi, S.Y. Graphene-based plasmonic waveguides for photonic integrated circuits. Opt. Express 2011, 19, 24557-24562. [CrossRef]

7. Anker, J.N.; Hall, W.P.; Lyandres, O.; Shah, N.C.; Zhao, J.; Van Duyne, R.P. Biosensing with plasmonic nanosensors. Nat. Mater. 2008, 7, 442-453. [CrossRef]

8. Ma, R.M.; Oulton, R.F.; Sorger, V.J.; Zhang, X. Plasmon lasers: Coherent light source at molecular scales. Laser Photonics Rev. 2012, 7, 1-21. [CrossRef]

9. Novoselov, K.S.; Geim, A.K.; Morozov, S.V.; Jiang, D.; Zhang, Y.; Dubonos, S.V.; Grigorieva, I.V.; Firsov, A.A. Electric field effect in atomically thin carbon films. Science 2004, 306, 666-669. [CrossRef]

10. Koppens, F.H.L.; Chang, D.E.; García de Abajo, F.J. Graphene plasmonics: A platform for strong light-matter interactions. Nano Lett. 2011, 11, 3370-3377. [CrossRef]

11. Argyropoulos, C. Enhanced transmission modulation based on dielectric metasurfaces loaded with graphene. Opt. Express 2015, 23, 23787-23797. [CrossRef] [PubMed]

12. Vakil, A.; Engheta, N. Transformation optics using graphene. Science 2011, 332, 1291-1294. [CrossRef] [PubMed]

13. Dai, Y.; Zhu, X.; Mortensen, N.A.; Zi, J.; Xiao, S. Nanofocusing in a tapered graphene plasmonic waveguide. J. Opt. 2015, 17, 065002. [CrossRef]

14. Liu, P.; Zhang, X.; Ma, Z.; Cai, W.; Wang, L.; Xu, J. Surface plasmon modes in graphene wedge and groove waveguides. Opt. Express 2013, 21, 32432-32440. [CrossRef] [PubMed]

15. Zhou, Y.; Zhu, Y.Y.; Zhang, K.; Wu, H.W.; Peng, R.W.; Fan, R.H.; Wang, M. Plasmonic band structures in doped graphene tubes. Opt. Express 2017, 25, 12081-12089. [CrossRef] [PubMed]

16. Chen, J.; Zeng, Y.; Xu, X.; Chen, X.; Zhou, Z.; Shi, P.; Yi, Z.; Ye, X.; Xiao, S.; Yi, Y. Plasmonic absorption enhancement in elliptical graphene arrays. Nanomaterials 2018, 8, 175. [CrossRef]

17. Ding, Y.; Guan, X.; Zhu, X.; Hu, H.; Bozhevolnyi, S.I.; Oxenløwe, L.K.; Jin, K.J.; Mortensen, N.A.; Xiao, S. Efficient electro-optic modulation in low-loss graphene-plasmonic slot waveguides. Nanoscale 2017, 9, 15576-15581. [CrossRef]

18. Gonçalves, P.A.D.; Dias, E.J.C.; Xiao, S.; Vasilevskiy, M.I.; Mortensen, N.A.; Peres, N.M.R. Graphene plasmons in triangular wedges and grooves. ACS Photonics 2016, 3, 2176-2183. [CrossRef]

19. Jabbarzadeh, F; Habibzadeh-Sharif, A. Double V-groove dielectric loaded plasmonic waveguide for sensing applications. J. Opt. Soc. Am. B 2019, 36, 690-696. [CrossRef]

20. Ansell, D.; Radko, I.P.; Han, Z.; Rodriguez, F.J.; Bozhevolnyi, S.I.; Grigorenko, A.N. Hybrid graphene plasmonic waveguide modulators. Nat. Commun. 2015, 6, 8846. [CrossRef]

21. Hao, R.; Jiao, J.; Peng, X.; Zhen, Z.; Dagarbek, R.; Zou, Y.; Li, E. Experimental demonstration of a graphene-based hybrid plasmonic modulator. Opt. Lett. 2019, 44, 2586-2589. [CrossRef] [PubMed]

22. Gubin, M.Y.; Leksin, A.Y.; Shesterikov, A.V.; Prokhorov, A.V.; Volkov, V.S. All-plasmonic switching effect in the graphene nanostructures containing quantum emitters. Nanomaterials 2020, 10, 122. [CrossRef]

23. Ono, M.; Hata, M.; Tsunekawa, M.; Nozaki, K.; Sumikura, H.; Chiba, H.; Notomi, M. Ultrafast and energy-efficient all-optical switching with graphene-loaded deep-subwavelength plasmonic waveguides. Nat. Photonics 2020, 14, 37-43. [CrossRef]

24. Guo, Z.; Nie, X.; Shen, F.; Zhou, H.; Zhou, Q.; Gao, J.; Guo, K. Actively tunable terahertz switches based on subwavelength graphene waveguide. Nanomaterials 2018, 8, 665. [CrossRef] [PubMed] 
25. Pei, C.; Yang, L.; Wang, G.; Wang, Y.; Jiang, X.; Hao, Y.; Li, Y.; Yang, J. Broadband graphene/glass hybrid waveguide polarizer. IEEE Photonics Technol. Lett. 2015, 27, 927-930. [CrossRef]

26. Hu, X.; Wang, J. Ultrabroadband compact graphene-silicon TM-pass polarizer. IEEE Photon. J. 2017, 9, 1-10. [CrossRef]

27. Wu, D.; Tian, J.; Li, L.; Yang, R. Plasmon induced transparency and refractive index sensing in a new type of graphene-based plasmonic waveguide. Opt. Commun. 2018, 412, 41-48. [CrossRef]

28. Luongo, G.; Giubileo, F.; Genovese, L.; Iemmo, L.; Martucciello, N.; Di Bartolomeo, A. I-V and C-V Characterization of a High-Responsivity Graphene/Silicon Photodiode with Embedded MOS Capacitor. Nanomaterials 2017, 7, 158. [CrossRef]

29. Hosseininejad, S.E.; Neshat, M.; Faraji-Dana, R.; Lemme, M.; Bolívar, P.H.; Cabellos-Aparicio, A.; Alarcón, E.; Abadal, $\mathrm{S}$. Reconfigurable THz plasmonic antenna based on few-layer graphene with high radiation efficiency. Nanomaterials 2018, 8, 577. [CrossRef]

30. Gao, Y.; Ren, G.; Zhu, B.; Liu, H.; Lian, Y.; Jian, S. Analytical model for plasmon modes in graphene-coated nanowire. Opt. Express 2014, 22, 24322-24331. [CrossRef]

31. Gao, Y.; Ren, G.; Zhu, B.; Wang, J.; Jian, S. Single-mode graphene-coated nanowire plasmonic waveguide. Opt. Lett. 2014, 39, 5909-5912. [CrossRef] [PubMed]

32. Kuzmin, D.A.; Bychkov, I.V.; Shavrov, V.G.; Temnov, V.V.; Lee, H.I.; Mok, J. Plasmonically induced magnetic field in graphene-coated nanowires. Opt. Lett. 2016, 41, 396-399. [CrossRef] [PubMed]

33. Teng, D.; Wang, K.; Li, Z.; Zhao, Y. Graphene-coated nanowire dimers for deep subwavelength waveguiding in mid-infrared range. Opt. Express 2019, 27, 12458-12469. [CrossRef]

34. Huang, Y.; Zhang, L.; Yin, H.; Zhang, M.; Su, H.; Li, I.L.; Liang, H. Graphene-coated nanowires with a drop-shaped cross section for $10 \mathrm{~nm}$ confinement and $1 \mathrm{~mm}$ propagation. Opt. Lett. 2017, 42, 2078-2081. [CrossRef] [PubMed]

35. Liu, J.P.; Zhai, X.; Wang, L.L.; Li, H.J.; Xie, F.; Lin, Q.; Xia, S.X. Analysis of mid-infrared surface plasmon modes in a graphene-based cylindrical hybrid waveguide. Plasmonics 2016, 11, 703-711. [CrossRef]

36. Heydari, M.B.; Samiei, M.H.V. Plasmonic graphene waveguides: A literature review. arXiv 2018, arXiv:1809.09937.

37. Zhang, Q.; Li, X.; Hossain, M.M.; Xue, Y.; Zhang, J.; Song, J.; Liu, J.; Turner, M.D.; Fan, S.; Bao, Q.; et al. Graphene surface plasmons at the near-infrared optical regime. Sci. Rep. 2014, 4, 6559. [CrossRef]

38. Cao, K.; Feng, S.; Han, Y.; Gao, L.; Ly, T.H.; Xu, Z.; Lu, Y. Elastic straining of free-standing monolayer graphene. Nat. Commun. 2020, 11, 1-7. [CrossRef]

39. Guo, M.; Qian, Y.; Qi, H.; Bi, K.; Chen, Y. Experimental measurements on the thermal conductivity of strained monolayer graphene. Carbon 2020, 157, 185-190. [CrossRef]

40. Jabbarzadeh, F.; Heydari, M.; Habibzadeh-Sharif, A. A comparative analysis of the accuracy of Kubo formulations for graphene plasmonics. Mater. Res. Exp. 2019, 6, 086209. [CrossRef]

41. Oulton, R.F.; Sorger, V.J.; Genov, D.A.; Pile, D.F.P.; Zhang, X. A hybrid plasmonic waveguide for subwavelength confinement and long-range propagation. Nat. Photonics 2008, 2, 496-500. [CrossRef]

42. Buckley, R.; Berini, P. Figures of merit for 2D surface plasmon waveguides and application to metal stripes. Opt. Express 2007, 15, 12174-12182. [CrossRef] [PubMed]

43. Yuan, Y.; Yao, J.; Xu, W. Terahertz photonic states in semiconductor-graphene cylinder structures. Opt. Lett. 2012, 37, 960-962. [CrossRef] [PubMed]

44. Zhao, J.; Liu, X.; Qiu, W.; Ma, Y.; Huang, Y.; Wang, J.-X.; Qiang, K.; Pan, J.-Q. Surface-plasmon-polariton whispering-gallery mode analysis of the graphene monolayer coated InGaAs nanowire cavity. Opt. Express 2014, 22, 5754-5761. [CrossRef] [PubMed]

45. Correas-Serrano, D.; Gomez-Diaz, J.S.; Alvarez-Melcon, A. Surface plasmons in graphene cylindrical waveguides. In Proceedings of the 2014 IEEE Antennas and Propagation Society International Symposium (APSURSI), Memphis, TN, USA, 6-11 July 2014.

46. Cao, T.; Tian, L.; Liang, H.; Qin, K.-R. Reconfigurable, graphene-coated, chalcogenide nanowires with a sub-10-nm enantioselective sorting capability. Microsyst. Nanoeng. 2018, 4, 7. [CrossRef] [PubMed]

47. Chen, B.; Meng, C.; Yang, Z.; Li, W.; Lin, S.; Gu, T.; Guo, X.; Wang, D.; Yu, S.; Wong, C.W.; et al. Graphene coated $\mathrm{ZnO}$ nanowire optical waveguides. Opt. Express 2014, 22, 24276-24285. [CrossRef]

48. He, X.; Zhang, X.; Zhang, H.; Xu, M. Graphene covered on microfiber exhibiting polarization and polarization-dependent saturable absorption. IEEE J. Sel. Top. Quant. 2014, 20, 55-61. 
49. Zhu, B.; Ren, G.; Gao, Y.; Yang, Y.; Lian, Y.; Jian, S. Graphene-coated tapered nanowire infrared probe: A comparison with metal-coated probes. Opt. Express 2014, 22, 24096-24103. [CrossRef]

50. Stockman, M.I. Nanofocusing of optical energy in tapered plasmonic waveguides. Phys. Rev. Lett. 2004, 93, 137404. [CrossRef]

51. Zhu, B.; Ren, G.; Gao, Y.; Yang, Y.; Wu, B.; Lian, Y.; Jian, S. Nanofocusing in the graphene-coated tapered nanowire infrared probe. J. Opt. Soc. Am. B 2015, 32, 955-960. [CrossRef]

52. Davoyan, A.R.; Engheta, N. Salient features of deeply subwavelength guiding of terahertz radiation in graphene-coated fibers. ACS Photonics 2016, 3, 737-742. [CrossRef]

53. Cong, X.; Huang, Y.; Zhang, M.; Su, H.; Li, I.L.; Liang, H. Graphene-coated nanowires with drop-shaped cross section for the low loss propagation of $\mathrm{THz}$ waves with sub-micron mode widths. Laser Phys. Lett. 2018, 15, 096001. [CrossRef]

54. Teng, D.; Wang, K.; Li, Z.; Zhao, Y.; Zhao, G.; Li, H.; Wang, H. Graphene-coated elliptical nanowires for low loss subwavelength terahertz transmission. Appl. Sci. 2019, 9, 2351. [CrossRef]

55. Cheng, X.; Xue, W.-R.; Wei, Z.-Z.; Dong, H.-Y.; Li, C.-Y. Mode characteristic analysis of optical waveguides based on graphene-coated elliptical dielectric nanowire. Acta Phys. Sin. 2019, 68, 058101.

56. Wu, Z.; Ning, T.; Li, J.; Zhang, M.; Su, H.; Li, I.L.; Liang, H. Tunable photonic-like modes in graphene-coated nanowires. Opt. Express 2019, 27, 35238-35244. [CrossRef] [PubMed]

57. Cuevas, M.; Riso, M.A.; Depine, R.A. Complex frequencies and field distributions of localized surface plasmon modes in graphene-coated subwavelength wires. J. Quant. Spectrosc. Radiat. Transf. 2016, 173, 26-33. [CrossRef]

58. Kuzmin, D.A.; Bychkov, I.V.; Shavrov, V.G.; Kotov, L.N. Transverse-electric plasmonic modes of cylindrical graphene-based waveguide at near-infrared and visible frequencies. Sci. Rep. 2016, 6, 26915. [CrossRef]

59. Liu, J.-P.; Zhai, X.; Xie, F.; Wang, L.-L.; Xia, S.-X.; Li, H.-J.; Luo, X.; Shang, X.-J. Analytical model of mid-infrared surface plasmon modes in a cylindrical long-range waveguide with double-layer graphene. J. Light. Technol. 2017, 35, 1971-1979. [CrossRef]

60. Zhao, T.; Hu, M.; Zhong, R.; Chen, X.; Zhang, P.; Gong, S.; Zhang, C.; Liu, S. Plasmon modes of circular cylindrical double-layer graphene. Opt. Exp. 2016, 24, 20461-20471. [CrossRef]

61. Cheng, X.; Xue, W.R.; Wei, Z.Z.; Dong, H.Y.; Li, C.Y. Mode analysis of a confocal elliptical dielectric nanowire coated with double-layer graphene. Opt. Commun. 2019, 452, 467-475. [CrossRef]

62. Correas-Serrano, D.; Gomez-Diaz, J.S.; Alu, A.; Alvarez-Melcon, A. Electrically and magnetically biased graphene-based cylindrical waveguides: Analysis and applications as reconfigurable antennas. IEEE Trans. Terahertz Sci. Technol. 2015, 5, 951-960. [CrossRef]

63. Mbonye, M.; Mendis, R.; Mittleman, D.M. A terahertz two-wire waveguide with low bending loss. Appl. Phys. Lett. 2009, 95, 233506. [CrossRef]

64. Gao, H.; Cao, Q.; Teng, D.; Zhu, M.; Wang, K. Perturbative solution for terahertz two-wire metallic waveguides with different radii. Opt. Express 2015, 23, 27457-27473. [CrossRef] [PubMed]

65. Zhu, B.; Ren, G.; Yang, Y.; Gao, Y.; Wu, B.; Lian, Y.; Wang, J.; Jian, S. Field enhancement and gradient force in the graphene-coated nanowire pairs. Plasmonics 2015, 10, 839-845. [CrossRef]

66. Zhai, L.; Xue, W.; Yang, R.; Han, L. Propagation properties of nano dielectric parallel lines coated with graphene. Acta Opt. Sin. 2015, 35, 1123002. [CrossRef]

67. Peng, Y.-L.; Xue, W.-R.; Wei, Z.-Z.; Li, C.-Y. Mode properties analysis of graphene-coated asymmetric parallel dielectric nanowire waveguides. Acta Phys. Sin. 2018, 67, 038102.

68. Wu, D.; Tian, J. Study on the plasmonic characteristics of bow-tie type graphene-coated nanowire pair. Optik 2018, 156, 689-695. [CrossRef]

69. Teng, D.; Wang, K.; Huan, Q.; Zhao, Y.; Tang, Y. High-performance transmission of surface plasmons in graphene-covered nanowire pairs with substrate. Nanomaterials 2019, 9, 1594. [CrossRef]

70. Raad, S.H.; Atlasbaf, Z.; Zapata-Rodríguez, C.J. Multi-frequency near-field enhancement with graphene-coated nano-disk homo-dimers. Opt. Express 2019, 27, 37012-37024. [CrossRef]

71. Kim, J.T.; Yu, Y.-J.; Choi, H.; Choi, C.-G. Graphene-based plasmonic photodetector for photonic integrated circuits. Opt. Express 2014, 22, 803-808. [CrossRef]

72. Hajati, M.; Hajati, Y. High-performance and low-loss plasmon waveguiding in graphene-coated nanowire with substrate. J. Opt. Soc. Am. B 2016, 33, 2560-2565. [CrossRef] 
73. Hajati, M.; Hajati, Y. Plasmonic Characteristics of two vertically coupled graphene-coated nanowires integrated with substrate. Appl. Opt. 2017, 56, 870-875. [CrossRef] [PubMed]

74. Wu, D.; Tian, J.; Yang, R. Study of mode performances of graphene-coated nanowire integrated with triangle wedge substrate. J. Nonlinear Opt. Phys. Mater. 2018, 27, 1850013. [CrossRef]

75. Sun, M.; Tian, J.; Lan, X.; He, Z.; Liu, J. Transmission properties of two vertically coupled double-graphene-coated nanowires integrated with substrate. Optik 2019, 185, 242-247. [CrossRef]

76. Wang, X.; Wang, J.; Liu, D.; Xia, X. Active manipulating propagation in the graphene hybrid plasmonic waveguides in mid-infrared region. In Electro-Optical and Infrared Systems: Technology and Applications XIII; SPIE: Edinburgh, UK, 2016.

77. Zhou, X.; Zhang, T.; Chen, L.; Hong, W.; Li, X. A graphene-based hybrid plasmonic waveguide with ultra-deep subwavelength confinement. J. Light. Technol. 2014, 32, 4199-4203. [CrossRef]

78. Liu, J.-P.; Zhai, X.; Wang, L.-L.; Li, H.-J.; Xie, F.; Xia, S.-X.; Shang, X.-J.; Luo, X. Graphene-based long-range SPP hybrid waveguide with ultra-long propagation length in mid-infrared range. Opt. Express 2016, 24 , 5376-5386. [CrossRef]

79. Chen, M.; Sheng, P.; Sun, W.; Cai, J. A symmetric terahertz graphene-based hybrid plasmonic waveguide. Opt. Commun. 2016, 376, 41-46. [CrossRef]

80. Ye, L.; Sui, K.; Liu, Y.; Zhang, M.; Liu, Q.H. Graphene-based hybrid plasmonic waveguide for highly efficient broadband mid-infrared propagation and modulation. Opt. Express 2018, 26, 15935-15947. [CrossRef]

81. Wang, Y.; Liu, H.; Wang, S.; Cai, M.; Ma, L. Optical transport properties of graphene surface plasmon polaritons in mid-infrared band. Crystals 2019, 9, 354. [CrossRef]

82. Cui, J.; Sun, Y.; Wang, L.; Ma, P. Graphene plasmonic waveguide based on a high-index dielectric wedge for compact photonic integration. Optik 2016, 127, 152-155. [CrossRef]

83. He, X.; Ning, T.; Lu, S.; Zheng, J.; Li, J.; Li, R.; Pei, L. Ultralow loss graphene-based hybrid plasmonic waveguide with deep-subwavelength confinement. Opt. Express 2018, 26, 10109-10118. [CrossRef] [PubMed]

84. He, X.Q.; Ning, T.G.; Pei, L.; Zheng, J.J.; Li, J.; Wen, X.D. Tunable hybridization of graphene plasmons and dielectric modes for highly confined light transmit at terahertz wavelength. Opt. Express 2019, 27, 5961-5972. [CrossRef] [PubMed]

85. He, X.; Ning, T.; Zheng, J.; Li, J.; Pei, L.; Wu, B. Deep-subwavelength light transmission in hybrid graphene-dielectric slot waveguide. J. Opt. 2019, 21, 095001. [CrossRef]

86. Wan, P.; Yang, C.; Liu, Z. Channel hybrid plasmonic modes in dielectric-loaded graphene groove waveguides. Opt. Commun. 2018, 420, 72-77. [CrossRef]

87. Molina, M.I.; Kivshar, Y.S. Discrete and surface solitons in photonic graphene nanoribbons. Opt. Lett. 2010, 35, 2895-2897. [CrossRef]

88. Kou, Y.; Förstner, J. Discrete plasmonic solitons in graphene-coated nanowire arrays. Opt. Express 2016, 24, 4714-4721. [CrossRef]

89. Meng, P.; Zhao, D.; Zhong, D.; Liu, W. Topological plasmonic modes in graphene-coated nanowire arrays. Opt. Quant. Electron. 2019, 51, 156. [CrossRef]

90. Wei, Z.-Z.; Xue, W.-R.; Peng, Y.-L.; Cheng, X.; Li, C.-Y. Mode characteristics of waveguides based on three graphene-coated dielectric nanowires. Acta Opt. Sin. 2019, 39, 0124001.

91. Wei, Z.-Z.; Xue, W.-R.; Peng, Y.-L.; Cheng, X.; Li, C.-Y. Modes characteristics analysis of THz waveguides based on three graphene-coated dielectric nanowires. Acta Phys. Sin. 2018, 67, 108101.

92. Ghaemi, H.F.; Thio, T.; Grupp, D.E.; Ebbesen, T.W.; Lezec, H.J. Surface plasmons enhance optical transmission through subwavelength holes. Phys. Rev. B 1998, 58, 6779-6782. [CrossRef]

93. Cao, Q.; Lalanne, P. Negative role of surface plasmons in the transmission of metallic gratings with very narrow slits. Phys. Rev. Lett. 2002, 88, 057403. [CrossRef]

94. Fei, Z.; Rodin, A.S.; Andreev, G.O.; Bao, W.; McLeod, A.S.; Wagner, M.; Zhang, L.M.; Zhao, Z.; Thiemens, M.; Dominguez, G.; et al. Gate-tuning of graphene plasmons revealed by infrared nano-imaging. Nature 2012, 487, 82-85. [CrossRef] [PubMed]

95. Chen, J.; Badioli, M.; Alonso-González, P.; Thongrattanasiri, S.; Huth, F.; Osmond, J.; Spasenović, M.; Centeno, A.; Pesquera, A.; Godignon, P.; et al. Optical nano-imaging of gate-tunable graphene plasmons. Nature 2012, 487, 77-81. [CrossRef] [PubMed]

96. Gao, W.; Shu, J.; Qiu, C.; Xu, Q. Excitation of plasmonic waves in graphene by guided-mode resonances. ACS Nano 2012, 6, 7806-7813. [CrossRef] [PubMed] 
97. Tao, J.; Dong, Z.; Yang, J.K.W.; Wang, Q.J. Plasmon excitation on flat graphene by s-polarized beams using four-wave mixing. Opt. Express 2015, 23, 7809-7819. [CrossRef]

98. Nikitin, A.Y.; Alonso-González, P.; Hillenbrand, R. Efficient coupling of light to graphene plasmons by compressing surface polaritons with tapered bulk materials. Nano Lett. 2014, 14, 2896-2901. [CrossRef]

99. Xia, S.-X.; Zhai, X.; Wang, L.-L.; Liu, J.-P.; Li, H.-J.; Liu, J.-Q.; Pan, A.-L.; Wen, S.-C. Excitation of surface plasmons in graphene-coated nanowire arrays. J. Appl. Phys. 2016, 120, 103104. [CrossRef]

100. Asgari, S.; Kashani, Z.G.; Granpayeh, N. Tunable nano-scale graphene-based devices in mid-infrared wavelengths composed of cylindrical resonators. J. Opt. 2018, 20, 045001. [CrossRef]

101. Cao, T.; Li, Y.; Tian, L.; Liang, H.; Qin, K. Fast switching "on/off" chiral surface plasmon polaritons in graphene-coated $\mathrm{Ge}_{2} \mathrm{Sb}_{2} \mathrm{Te}_{5}$ nanowire. ACS Appl. Nano Mater. 2018, 1, 759-767. [CrossRef]

102. Gan, X.; Zhao, C.; Wang, Y.; Mao, D.; Fang, L.; Han, L.; Zhao, J. Graphene-assisted all-fiber phase shifter and switching. Optica 2015, 2, 468-471. [CrossRef]

103. Jalas, D.; Petrov, A.; Eich, M.; Freude, W.; Fan, S.; Yu, Z.; Baets, R.; Popović, M.; Melloni, A.; Joannopoulos, J.D.; et al. What is-And what is not-An optical isolator. Nat. Photonics 2013, 7, 579-582. [CrossRef]

104. Pae, J.-S.; Im, S.-J.; Ri, C.-S.; Ho, K.-S.; Song, G.-S.; Han, Y.-H.; Herrmann, J. Magnetoplasmonic isolators based on graphene waveguide ring resonators. Phys. Rev. B 2019, 100, 041405. [CrossRef]

105. Kou, J.; Chen, J.; Chen, Y.; Xu, F.; Lu, Y. Platform for enhanced light-graphene interaction length and miniaturizing fiber stereo devices. Optica 2014, 1, 307-310. [CrossRef]

106. Liu, M.; Yin, X.; Ulin-Avila, E.; Geng, B.; Zentgraf, T.; Ju, L.; Wang, F.; Zhang, X. A graphene-based broadband optical modulator. Nature 2011, 474, 64-67. [CrossRef]

107. Li, W.; Chen, B.; Meng, C.; Fang, W.; Xiao, Y.; Li, X.; Hu, Z.; Xu, Y.; Tong, L.; Wang, H.; et al. Ultrafast all-optical graphene modulator. Nano Lett. 2014, 14, 955-959. [CrossRef]

108. Chen, J.-H.; Zheng, B.-C.; Shao, G.-H.; Ge, S.-J.; Xu, F.; Lu, Y.-Q. An all-optical modulator based on a stereo graphene-microfiber structure. Light Sci. Appl. 2015, 4, e360. [CrossRef]

109. Liang, H.; Zhang, L.; Zhang, S.; Cao, T.; Alù, A.; Ruan, S.; Qiu, C.-W. Gate-programmable electro-optical addressing array of graphene-coated nanowires with sub-10 nm resolution. ACS Photonics 2016, 3, 1847-1853. [CrossRef]

110. He, X.; Liu, Z.-B.; Wang, D.; Yang, M.; Liao, C.R.; Zhao, X. Passively mode-locked fiber laser based on reduced graphene oxide on microfiber for ultra-wide-band doublet pulse generation. J. Light. Technol. 2012, 30, 984-989. [CrossRef]

111. Zhao, N.; Liu, M.; Liu, H.; Zheng, X.-W.; Ning, Q.-Y.; Luo, A.-P.; Luo, Z.-C.; Xu, W.-C. Dual-wavelength rectangular pulse $\mathrm{Yb}$-doped fiber laser using a microfiber-based graphene saturable absorber. Opt. Express 2014, 22, 10906-10913. [CrossRef]

112. Liu, X.M.; Yang, H.R.; Cui, Y.D.; Chen, G.W.; Yang, Y.; Wu, X.Q.; Yao, X.K.; Han, D.D.; Han, X.X.; Zeng, C.; et al. Graphene-clad microfibre saturable absorber for ultrafast fibre lasers. Sci. Rep. 2016, 6, 26024. [CrossRef]

113. Yao, B.C.; Rao, Y.J.; Huang, S.W.; Wu, Y.; Feng, Z.Y.; Choi, C.; Liu, H.; Qi, H.F.; Duan, X.F.; Peng, G.D.; et al. Graphene Q-switched distributed feedback fiber lasers with narrow linewidth approaching the transform limit. Opt. Express 2017, 25, 8202-8211. [CrossRef] [PubMed]

114. Wang, X.-F.; Zhang, J.-H.; Peng, X.-L.; Mao, X.-F. Generation and evolution of multiple operation states in passively mode-locked thulium-doped fiber laser by using a graphene-covered-microfiber. Chin. Phys. $B$ 2018, 27, 084215. [CrossRef]

115. Li, D.; Zhu, J.; Jiang, M.; Li, D.; Wu, H.; Han, J.; Sun, Z.; Ren, Z. Active-passive Q-switched fiber laser based on graphene microfiber. Appl. Phys. B 2019, 125, 203. [CrossRef]

116. Almeida, V.R.; Lipson, M. Optical bistability on a silicon chip. Opt. Lett. 2004, 29, 2387-2389. [CrossRef] [PubMed]

117. Li, R.; Wang, H.; Zheng, B.; Dehdashti, S.; Li, E.; Chen, H. Bistable scattering in graphene-coated dielectric nanowires. Nanoscale 2017, 9, 8449-8457. [CrossRef] [PubMed]

118. Zhang, K.; Gao, L. Optical bistability in graphene-wrapped dielectric nanowires. Opt. Express 2017, 25, 13747-13759. [CrossRef]

119. Feng, Q.-Y.; Yao, B.-C.; Zhou, J.-H.; Xia, H.-D.; Fan, M.-Q.; Zhang, L.; Wu, Y.; Rao, Y.-J. Four-wave-mixing generated by femto-second laser pumping based on graphene coated microfiber structure. Acta Phys. Sin. $2015,64,184214$. 
120. Gao, Y.; Shadrivov, I.V. Second harmonic generation in graphene-coated nanowires. Opt. Lett. 2016, 41, 3623-3626. [CrossRef]

121. Gao, Y.; Shadrivov, I.V. Nonlinear coupling in graphene-coated nanowires. Sci. Rep. 2016, 6, 38924. [CrossRef]

122. Kuzmin, D.A.; Bychkov, I.V.; Shavrov, V.G.; Temnov, V.V. Giant faraday rotation of high-order plasmonic modes in graphene-covered nanowires. Nano Lett. 2016, 16, 4391-4395. [CrossRef]

123. Cai, W.; Chettiar, U.K.; Kildishev, A.V.; Shalaev, V.M. Optical cloaking with metamaterials. Nat. Photonics 2007, 1, 224-227. [CrossRef]

124. Leonhardt, U. Optical conformal mapping. Science 2006, 312, 1777-1780. [CrossRef] [PubMed]

125. Chen, H.; Chan, C.T.; Sheng, P. Transformation optics and metamaterials. Nat. Mater. 2010, 9, $387-396$. [CrossRef] [PubMed]

126. Chen, P.-Y.; Alù, A. Atomically thin surface cloak using graphene monolayers. ACS Nano 2011, 5, 5855-5863. [CrossRef]

127. Naserpour, M.; Zapata-Rodríguez, C.J.; Vuković, S.M.; Pashaeiadl, H.; Belić, M.R. Tunable invisibility cloaking by using isolated graphene-coated nanowires and dimers. Sci. Rep. 2017, 7, 12186. [CrossRef]

128. Fesenko, V.I.; Shcherbinin, V.I.; Tuz, V.R. Multiple invisibility regions induced by symmetry breaking in a trimer of subwavelength graphene-coated nanowires. J. Opt. Soc. Am. A 2018, 35, 1760-1768. [CrossRef]

129. Shcherbinin, V.I.; Moskvitina, Y.K.; Fesenko, V.I.; Tuz, V.R. Dual-polarized all-angle cloaking of a dielectric nanowire by helical graphene ribbons. Phys. Rev. B 2019, 100, 035428. [CrossRef]

130. Jiang, J.; Zhang, D.; Zhang, B.; Luo, Y. Interaction between graphene-coated nanowires revisited with transformation optics. Opt. Lett. 2017, 42, 2890-2893. [CrossRef]

131. Yang, Y.; Shi, Z.; Li, J.; Li, Z.-Y. Optical forces exerted on a graphene-coated dielectric particle by a focused Gaussian beam. Photon. Res. 2016, 4, 65-69. [CrossRef]

132. Wei, B.; Jian, S. Multiple modes plasmon-induced-transparency and slow light effect in a compact graphene coated nanowire waveguide system. Opt. Commun. 2017, 402, 66-72. [CrossRef]

133. Arruda, T.J.; Bachelard, R.; Weiner, J.; Courteille, P.W. Tunable Fano resonances in the decay rates of a pointlike emitter near a graphene-coated nanowire. Phys. Rev. B 2018, 98, 245419. [CrossRef]

134. Olivo, J.; Cuevas, M. Enhanced energy transfer via graphene-coated wire surface plasmons. J. Quant. Spectrosc. Radiat. Transf. 2019, 239, 106655. [CrossRef]

(C) 2020 by the authors. Licensee MDPI, Basel, Switzerland. This article is an open access article distributed under the terms and conditions of the Creative Commons Attribution (CC BY) license (http://creativecommons.org/licenses/by/4.0/). 